\title{
PAPER
}

\section{Wigner-Smith matrix, exponential functional of the matrix Brownian motion and matrix Dufresne identity}

To cite this article: Aurélien Grabsch and Christophe Texier 2020 J. Phys. A: Math. Theor. 53425003

View the article online for updates and enhancements.

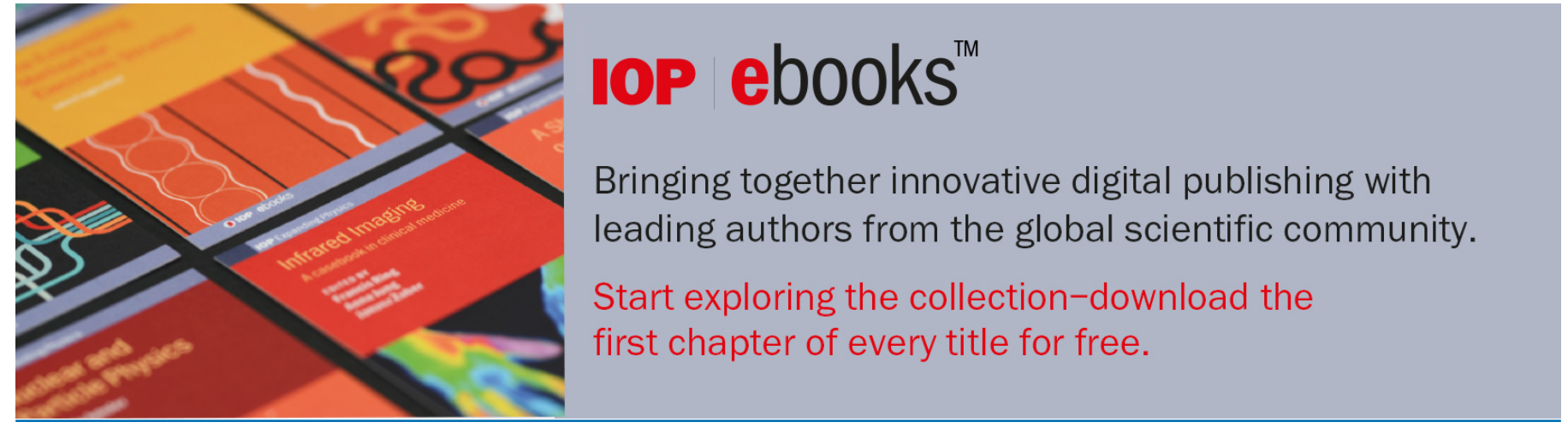

This content was downloaded from IP address 132.229 .211 .20 on 17/12/2020 at 19:24 


\title{
Wigner-Smith matrix, exponential functional of the matrix Brownian motion and matrix Dufresne identity
}

\author{
Aurélien Grabsch ${ }^{1} \odot$ and Christophe Texier $^{2} \odot$ \\ ${ }^{1}$ Instituut-Lorentz, Universiteit Leiden, PO Box 9506, 2300 RA, Leiden, The \\ Netherlands \\ 2 LPTMS, CNRS, Université Paris-Saclay, 91405 Orsay Cedex, France \\ E-mail: christophe.texier@u-psud.fr
}

Received 3 March 2020, revised 25 June 2020

Accepted for publication 2 July 2020

Published 2 October 2020

\begin{abstract}
We consider a multichannel wire with a disordered region of length $L$ and a reflecting boundary. The reflection of a wave of frequency $\omega$ is described by the scattering matrix $\mathcal{S}(\omega)$, encoding the probability amplitudes to be scattered from one channel to another. The Wigner-Smith time delay matrix $\mathcal{Q}=-\mathrm{i} \mathcal{S}^{\dagger} \partial_{\omega} \mathcal{S}$ is another important matrix, which encodes temporal aspects of the scattering process. In order to study its statistical properties, we split the scattering matrix in terms of two unitary matrices, $\mathcal{S}=\mathrm{e}^{2 \mathrm{i} k L} \mathcal{U}_{L} \mathcal{U}_{R}$ (with $\mathcal{U}_{L}=\mathcal{U}_{R}^{\mathrm{T}}$ in the presence of time reversal symmetry), and introduce a novel symmetrisation procedure for the Wigner-Smith matrix: $\widetilde{\mathcal{Q}}=\mathcal{U}_{R} \mathcal{Q} \mathcal{U}_{R}^{\dagger}=(2 L / v) \mathbf{1}_{N}-\mathrm{i} \mathcal{U}_{L}^{\dagger} \partial_{\omega}\left(\mathcal{U}_{L} \mathcal{U}_{R}\right) \mathcal{U}_{R}^{\dagger}$, where $k$ is the wave vector and $v$ the group velocity. We demonstrate that $\widetilde{\mathcal{Q}}$ can be expressed under the form of an exponential functional of a matrix Brownian motion. For semi-infinite wires, $L \rightarrow \infty$, using a matricial extension of the Dufresne identity, we recover straightforwardly the joint distribution for $\mathcal{Q}$ 's eigenvalues of Brouwer and Beenakker (2001 Physica E 9 463). For finite length $L$, the exponential functional representation is used to calculate the first moments $\langle\operatorname{tr}(\mathcal{Q})\rangle,\left\langle\operatorname{tr}\left(\mathcal{Q}^{2}\right)\right\rangle$ and $\left\langle[\operatorname{tr}(\mathcal{Q})]^{2}\right\rangle$. Finally we derive a partial differential equation for the resolvent $g(z ; L)=\lim _{N \rightarrow \infty}(1 / N) \operatorname{tr}\left\{\left(z \mathbf{1}_{N}-N \mathcal{Q}\right)^{-1}\right\}$ in the large $N$ limit.
\end{abstract}

Keywords: disordered systems, random matrices, stochastic processes

\section{Introduction}

Scattering of waves in complex media has been the subject of intense investigations for several decades, with applications in many areas of physics, ranging from compound-nucleus reactions 
[1, 2], chaotic billiards [3], electromagnetic waves in random media [4] to coherent electronic transport $[5,6]$. When the wave is elastically scattered by the static potential, the scattering process is encoded in the on-shell scattering matrix, with elements $\mathcal{S}_{a b}(\omega)$ characterizing the amplitude of the wave in the scattering channel $a$, if a wave of frequency $\omega$ was injected in channel $b$ (channels can be the open transverse modes of some wave guides). Given the $N \times N$ scattering matrix $\mathcal{S}$ as a function of the frequency, it is possible to construct another important matrix, known as the Wigner-Smith time delay matrix $[7,8]$

$$
\mathcal{Q}=-\mathrm{i} \mathcal{S}^{\dagger} \partial_{\omega} \mathcal{S}
$$

encoding several sets of times characterizing the scattering process (see references [9, 10] and references therein for a recent review of these concepts).

In complex media, it is natural to investigate the statistical properties of these two matrices (their sample to sample fluctuations). The most studied setting is a chaotic cavity, i.e. a zerodimensional situation. In such devices, the wave is injected through wave guides. The complex nature of the dynamics inside the cavity naturally leads to a random matrix formulation. Such a formulation can be justified from various microscopic or phenomenological models [11, 12], semiclassics [13] and also from some maximum entropy principle [6, 14]. For example, assuming perfect contacts, it is natural to assume that $\mathcal{S}$ belongs to one of the circular ensembles (COE, CUE or CSE), depending on the presence or absence of time reversal symmetry and/or spin rotational symmetry [15]. Based on such assumptions, with additional modelling of the frequency dependence [16], the distribution of the Wigner-Smith matrix eigenvalues for chaotic cavities has been obtained by Brouwer, Frahm and Beenakker (BFB) [17, 18]. In order to deal with a matrix statistically independent from the scattering matrix, BFB introduced the symmetrised Wigner-Smith matrix

$$
\mathcal{Q}_{s}=-\mathrm{i} \mathcal{S}^{-1 / 2} \frac{\partial \mathcal{S}}{\partial \omega} \mathcal{S}^{-1 / 2}
$$

Its inverse $\Gamma=\tau_{\mathrm{H}} \mathcal{Q}_{s}^{-1}$, where $\tau_{\mathrm{H}}$ is the Heisenberg time ${ }^{3}$, was shown to be distributed according to a specific instance of the Laguerre ensemble of random matrix theory, $P(\Gamma) \propto$ $(\operatorname{det} \Gamma)^{\beta N / 2} \mathrm{e}^{-(\beta / 2) \operatorname{tr}\{\Gamma\}}$, where the distribution is defined over the set of Hermitian matrices with positive eigenvalues. $\beta$ is the Dyson index $(\beta=1$ when time reversal symmetry holds and $\beta=2$ if not). Based on this distribution, many results have been obtained for ideal contacts: cumulants [19] and distribution [20] of its trace $\operatorname{tr}\{\mathcal{Q}\}$, or other correlations [21-25] (see the updated preprint version of reference [10] for an exhaustive review). Several generalizations of BFB's distribution have been obtained more recently: the case of non-ideal contacts has been studied [26, 27], BdG symmetry classes [26] and the effect of absorption (for ideal contacts) [28].

Several results are also known beyond the zero-dimensional case. The case of a strictly onedimensional disordered wire of length $L$ with a reflecting boundary, corresponding to $N=1$ scattering channel, is best understood. In this case the wave is expected to be localised by the disorder, on a typical scale $\xi$ (the localisation length). A wave packet may remain trapped a long time if the localisation centre is far from the edge of the disordered region, which gives rise to

\footnotetext{
${ }^{3} \tau_{\mathrm{H}}=2 \pi / \delta \omega$ where $\delta \omega$ is the mean level spacing between eigenmodes of the cavity.
} 
narrow resonances. In the high energy/weak disorder regime, when universality is expected, the relation to universality of localisation properties was established, which has led to a representation of the Wigner time delay under the form of the exponential functional of the Brownian motion (BM) [29] ${ }^{4}$

$$
\mathcal{Q} \stackrel{(\text { law })}{=} 2 \tau_{\xi} \int_{0}^{L / \xi} \mathrm{d} x \mathrm{e}^{-2 x+2 B(x)}
$$

where $B(x)$ is a normalised Brownian motion, such that $\langle B(x)\rangle=0$ and $\left\langle B(x) B\left(x^{\prime}\right)\right\rangle=$ $\min \left(x, x^{\prime}\right)$ (in references [30, 31] a different form, although equivalent to (3), was proposed). The characteristic scale

$$
\tau_{\xi}=\xi / v
$$

is the time needed by the particle with group velocity $v$ to cover the localisation length $\xi$. Using known results on exponential functionals of the BM [32, 33], the representation (3) has allowed to derive the moments $[29,31]$ of $\mathcal{Q}$ and its full distribution $P_{L}(\tau)$ [31] for finite $L$. The limit law of the Wigner time delay, for $L \rightarrow \infty$, was derived in references [29, 31] within a continuous model and later in [34] within a tight binding discrete model ${ }^{5}$

$$
P_{\infty}(\tau)=\frac{\tau_{\xi}}{\tau^{2}} \mathrm{e}^{-\tau_{\xi} / \tau}
$$

The exponential functional of the Brownian motion

$$
Z_{L}^{(\mu)}=\int_{0}^{L} \mathrm{~d} x \lambda(x)^{2} \quad \text { with } \lambda(x)=\mathrm{e}^{-\mu x+B(x)}
$$

with other functionals have attracted a considerable interest in the mathematical community [35-37]; the relation with several physical problems is reviewed in references [38, 39]. They have also found several applications in mathematical finance, in the context of which Dufresne has obtained the remarkable identity [40]

$$
Z_{\infty}^{(\mu)} \stackrel{\text { (law) }}{=} \frac{1}{\gamma^{(\mu)}} \quad \text { for } \mu>0
$$

where $\gamma^{(\mu)}$ obeys the Gamma-law

$$
p(\gamma)=\frac{1}{2^{\mu} \Gamma(\mu)} \gamma^{\mu-1} \mathrm{e}^{-\gamma / 2}
$$

The representation (6) makes clear that (3) corresponds to a drift $\mu=1$. Hence the limit law (5) is a direct consequence of the Dufresne identity (7).

Beyond the weak disorder/high energy universal regime in one dimension, some other results have been obtained. In the strictly one dimensional case, various results were also derived in the low energy/strong disorder regime [29, 41]. ${ }^{6}$ The case of higher dimensions

\footnotetext{
${ }^{4}$ An identity in law relates two quantities with same statistical properties. For example, the well-known scaling property of the Brownian motion can be written $B(\lambda x) \stackrel{(\text { law })}{=} \sqrt{\lambda} B(x)$.

${ }^{5}$ See the arXiv version of reference [10] for a detailed review.

${ }^{6}$ A non-trivial distribution for the time delay for the dimer model with delocalisation points [42] was also obtained in chapter 6 of [41].
} 
has also been investigated [43] (see the review article [44]). More recently, the marginal distribution of the proper time delays was studied by Ossipov [45], claiming to describe also the metallic regime in $d>2$; we criticize this statement at the end of the paper.

Another interesting case, which is more tractable, is the intermediate situation of quasione-dimensional systems, i.e. multichannel disordered wires. The assumption that channels are statistically equivalent (isotropy) allows to derive analytical results, such as the Lyapunov spectrum or the statistics of transmission probabilities [5]. The joint distribution of eigenvalues of $\Gamma=\tau_{\xi} \mathcal{Q}^{-1}$ has been derived for a semi-infinite disordered wire in references [46, 47]

$$
\mathcal{P}_{N}\left(\gamma_{1}, \ldots, \gamma_{N}\right) \propto \prod_{i<j}\left|\gamma_{i}-\gamma_{j}\right|^{\beta} \prod_{n} \mathrm{e}^{-\gamma_{n} / 2} \quad \text { for } L \rightarrow \infty,
$$

corresponding to the matrix distribution

$$
P(\Gamma) \propto \mathrm{e}^{-(1 / 2) \operatorname{tr}\{\Gamma\}},
$$

defined over the set of Hermitian matrices with positive eigenvalues. For $N=1$, the distribution corresponds to (5). This is a different instance of the Laguerre ensemble of random matrix theory than the one obtained for chaotic quantum dots and recalled above, after equation (2). This result has been used to show that the distribution of the Wigner time delay, i.e. the trace $\tau_{\mathrm{W}}=(1 / N) \operatorname{tr}\{\mathcal{Q}\}$, becomes independent of $N$ in the large $N$ limit [48]:

$$
\mathscr{P}_{N}^{(\beta)}(\tau) \simeq \frac{A_{\beta}}{\tau^{2}} \exp \left\{-\frac{27 \tau_{\xi}^{2}}{64 \beta \tau^{2}}+\left(\frac{2}{\beta}-1\right) \frac{9(2-\sqrt{3}) \tau_{\xi}}{8 \tau}\right\} \quad \text { for } L \rightarrow \infty
$$

where $A_{\beta}$ is a normalisation. This shows in particular that, as in the $N=1$ channel case, all moments $\left\langle\operatorname{tr}\{\mathcal{Q}\}^{n}\right\rangle$ are infinite for $L \rightarrow \infty$. The physical origin of the divergence lies in the proliferation of very narrow resonances (this is discussed for the case $N=1$ in reference [29]). Much less is known for finite length $L$. Using the fact that $N \tau_{\mathrm{W}} /(2 \pi)=(2 \pi)^{-1} \operatorname{tr}\{\mathcal{Q}\}$ can be interpreted as the density of states of the open system (see appendix A or reference [10]), for weak disorder, we can write

$$
\langle\operatorname{tr}\{\mathcal{Q}\}\rangle \simeq \frac{N L}{k}
$$

Nevertheless the behaviour of higher moments is an open question. It is the aim of the present article to study this problem and provide some statistical information on the Wigner-Smith time delay matrix for disordered wire of finite length $L$. For this purpose, we will obtain a generalisation of the representation (3), for $N>1$. In particular, this will provide a straightforward derivation of the distribution (9) for $L \rightarrow \infty$, by using an extension of the Dufresne identity (7) to the multichannel case, when $\mathcal{Q}$ is an $N \times N$ random matrix. Furthermore, this will allow to calculate the moments.

\subsection{The model of multichannel disordered wires}

Various models of multichannel disordered wires have been considered in the past. Dorokhov introduced a first microscopic model in reference [49] describing $N$ one-dimensional wires with independent scalar random potentials and uniform couplings between neighbouring wires. In reference [50], he analysed a different model for 1D chains subject to a random potential with a matrix structure, i.e a model where the couplings between chains is random. A more phenomenological scattering approach was followed by several authors (for a pedagogical presentation of this approach and a review see reference [5]). 
Here, we are interested in universal properties of multichannel disordered wires, thus the details of the model are not expected to be of importance. We have found convenient to study the multichannel generalization of the Halperin model with a Gaussian white noise potential [51]: the Schrödinger equation

$$
H \psi(x)=\varepsilon \psi(x) \text { with } H=-\mathbf{1}_{N} \partial_{x}^{2}+V(x),
$$

where $\psi(x)$ is a column vector with $N$ components, coupled by the potential $V(x)$. We consider the case where $V(x)$ is an $N \times N$ matrix Gaussian white noise with zero mean and correlations

$$
\left\langle V_{a b}(x) V_{c d}^{*}\left(x^{\prime}\right)\right\rangle=\sigma C_{a b, c d} \delta\left(x-x^{\prime}\right),
$$

where $\sigma$ is the disorder strength (with dimension $[\sigma]=L^{-3}$ ). We will assume isotropy among the channels, i.e. the invariance of the statistical properties of $V(x)$ under orthogonal $(\beta=1)$ or unitary $(\beta=2)$ transformations. For $\beta=1$ this is equivalent to the model of reference [50]. This leads to the correlations between channels ${ }^{7}$

$$
C_{a b, c d}=\frac{\beta}{2} \delta_{a c} \delta_{b d}+\left(1-\frac{\beta}{2}\right) \delta_{a d} \delta_{b c}= \begin{cases}\frac{1}{2}\left(\delta_{a c} \delta_{b d}+\delta_{a d} \delta_{b c}\right) & \text { for } \beta=1(\mathrm{TRS}), \\ \delta_{a c} \delta_{b d} & \text { for } \beta=2(\text { no TRS) }\end{cases}
$$

An important scale of the problem is the elastic scattering rate $1 / \tau_{\mathrm{e}}$, related to the self energy $\Sigma_{a b}^{\mathrm{R}}$ by [52]:

$$
\frac{1}{2 \tau_{\mathrm{e}}}=-\operatorname{Im} \Sigma_{a a}^{\mathrm{R}}
$$

Introducing the free retarded Green's function $G_{a, b}^{\mathrm{R}}\left(x, x^{\prime}\right)=\delta_{a, b} \frac{1}{2 \mathrm{i} k} \mathrm{e}^{\mathrm{i} k\left|x-x^{\prime}\right|}$ for energy $\varepsilon=k^{2}$, we get the perturbative expression of the self energy, at lowest order in the disorder,

$$
\Sigma_{a b}^{\mathrm{R}} \simeq \sum_{c} \sigma C_{a c, b c} G_{c, c}^{\mathrm{R}}(0,0)=-\frac{\mathrm{i} \sigma}{2 k} \sum_{c} C_{a c, b c}
$$

We deduce the elastic mean free path $\ell_{\mathrm{e}}=v \tau_{\mathrm{e}}$, where $v=\partial \varepsilon / \partial k=2 k$ is the group velocity, in terms of the disorder strength $\sigma$

$$
\ell_{\mathrm{e}} \simeq \frac{2 k^{2}}{\mu \sigma}
$$

where

$$
\mu=\sum_{b} C_{a b, a b}=1+\frac{\beta}{2}(N-1)
$$

As we will see, for weak disorder $\varepsilon=k^{2} \gg \sigma^{2 / 3}$, the localisation length is given by

$$
\xi \simeq \frac{8 k^{2}}{\sigma} \simeq 4 \mu \ell_{\mathrm{e}}=2[2+\beta(N-1)] \ell_{\mathrm{e}}
$$

which is the well-known dependence in $\beta$ and $N$ (obtained within the DMPK approach for a completely different model in reference [5]).

${ }^{7}(14)$ and (15) correspond to the distribution $P[V(x)]=\exp \left(-\frac{1}{2 \sigma} \int \mathrm{d} x \operatorname{tr}\left\{V(x)^{2}\right\}\right)$. 
Below, we study the scattering problem on $\mathbb{R}^{+}$for a potential defined on $[0, L]$ (and vanishing outside the interval). The eigenstate corresponding to inject the wave in channel $b \in\{1, \ldots, N\}$ is an $N$-component vector denoted $\psi_{\varepsilon}^{(b)}(x)$. We write the $a$ th component, i.e. the amplitude in channel $a$, in the free region as

$$
\left[\psi_{\varepsilon}^{(b)}(x)\right]_{a}=\frac{1}{\sqrt{h v}}\left(\delta_{a b} \mathrm{e}^{-\mathrm{i} k(x-L)}+\mathcal{S}_{a b}(\varepsilon) \mathrm{e}^{\mathrm{i} k(x-L)}\right) \quad \text { for } x \geqslant L .
$$

The prefactor ensures the normalisation [53] $\left\langle\psi_{\varepsilon}^{(a)} \mid \psi_{\varepsilon^{\prime}}^{(b)}\right\rangle=\delta_{a b} \delta\left(\varepsilon-\varepsilon^{\prime}\right)$.

\subsection{Statement of the main results}

Our analysis is based on a new symmetrization procedure of the Wigner-Smith matrix. Assuming that all channels are controlled by the same wave vector $k$ in the absence of disorder, we extract rapid oscillations of the scattering matrix $\mathcal{S}=\widetilde{\mathcal{S}} \mathrm{e}^{2 \mathrm{i} k L}$, where $\widetilde{\mathcal{S}}$ is controlled by slow variables. We use a 'square root trick' in order to decompose it in terms of two unitary matrices $\widetilde{\mathcal{S}}=\mathcal{U}_{L} \mathcal{U}_{R}$. In the presence of TRS $(\beta=1), \mathcal{U}_{L}=\mathcal{U}_{R}^{\mathrm{T}}$ ensures the property $\widetilde{\mathcal{S}}=\widetilde{\mathcal{S}}^{\mathrm{T}}$. In the absence of TRS $(\beta=2)$, they are chosen such that they obey two matrix stochastic differential equations (SDE) of convenient form. Then, the Wigner-Smith matrix is symmetrised as

$$
\widetilde{\mathcal{Q}}=\mathcal{U}_{R} \mathcal{Q} \mathcal{U}_{R}^{\dagger}=\frac{2 L}{v} \mathbf{1}_{N}-\mathrm{i} \mathcal{U}_{L}^{\dagger} \partial_{\varepsilon}\left(\mathcal{U}_{L} \mathcal{U}_{R}\right) \mathcal{U}_{R}^{\dagger}
$$

where $v$ is the group velocity. The first term is the result in the absence of the disorder: $2 L / v$ is the time needed to go back and forth in the sample when $V=0$. Our analysis relies on the decoupling between fast and slow variables in the high energy/weak disorder regime and on an isotropy assumption (invariance under exchange of channels). One of our main result is the matrix SDE

$$
\frac{\partial}{\partial L} \widetilde{\mathcal{Q}}=\frac{\mathbf{1}_{N}}{k}-\frac{2 \mu}{\xi} \widetilde{\mathcal{Q}}+\frac{1}{\sqrt{\xi}}(\widetilde{\mathcal{Q}} \eta(L)+\eta(L) \widetilde{\mathcal{Q}}) \quad(\text { Stratonovich })
$$

for $\mu=1+\frac{\beta}{2}(N-1)$ and where $\eta(x)$ is a normalised Hermitian Gaussian white noise, $\left\langle\eta_{a b}(x) \eta_{c d}^{*}\left(x^{\prime}\right)\right\rangle=C_{a b, c d} \delta\left(x-x^{\prime}\right)$ with (15). From this matrix SDE, we deduce a representation of the Wigner-Smith time delay matrix under the form of an exponential functional of a matrix Brownian motion

$$
\widetilde{\mathcal{Q}} \stackrel{\text { (law) }}{=} 2 \tau_{\xi} \int_{0}^{L / \xi} \mathrm{d} x \Lambda(x)^{\dagger} \Lambda(x)
$$

where $\Lambda(x)$ obeys the matrix SDE

$$
\partial_{x} \Lambda(x)=-\mu \Lambda(x)+\eta(x) \Lambda(x) \quad \text { (Stratonovich), }
$$

with $\Lambda(0)=\mathbf{1}_{N}$. We may also write

$$
\Lambda(x)=T \mathrm{e}^{-\mu x+\int_{0}^{x} \mathrm{~d} t \eta(t)}
$$

where $T$ denotes chronological ordering, to make the contact with formulae (3) and (6) more explicit. For $N=1$ channel, we recover (3) (i.e. the form (6) for $\mu=1$ ). The representation (22) has allowed us to recover straightforwardly the result of Beenakker and Brouwer (9), by using a matrix generalization of the Dufresne identity

$$
\widetilde{\mathcal{Q}} \stackrel{\text { (law) }}{=} 2 \tau_{\xi} \Gamma^{-1} \quad \text { for } L \rightarrow \infty
$$


where $\Gamma$ obeys the Laguerre distribution (10).

As an application of the matrix SDE for $\widetilde{\mathcal{Q}}$, we show on the example of $\langle\operatorname{tr}\{\mathcal{Q}\}\rangle,\left\langle\operatorname{tr}\{\mathcal{Q}\}^{2}\right\rangle$ and $\left\langle\operatorname{tr}\left\{\mathcal{Q}^{2}\right\}\right\rangle$, how moments can be computed.

Finally, we reconsider the problem studied by Ossipov [45], within our model based on isotropy assumption. We recover Ossipov's equation for the resolvent $g(z ; L)=$ $\lim _{N \rightarrow \infty}(1 / N) \operatorname{tr}\left\{\left[z \mathbf{1}_{N}-N \mathcal{Q} /\left(2 \tau_{\xi}\right)\right]^{-1}\right\}$, which casts doubts on Ossipov's claim to describe the metallic phase in dimension $d>2$, as our model describes disordered wires transversally ergodic.

\subsection{Outline}

In section 2, starting from a representation of the Wigner-Smith matrix in terms of the wave function, we show that localisation properties in multichannel disordered wires explain the origin of the relation with exponential functional of the matrix Brownian motion. The following sections are devoted to a more precise derivation of this relation, with no prior knowledge of the localisation properties. The analysis is based on the study of matrix stochastic differential equations (MSDE): the main SDE are derived section 3. Then, section 4 discusses the elimination of the fast variables in the high energy regime, leading to new MSDE for slow variables. A new symmetrisation procedure of the Wigner-Smith matrix is introduced in section 5. The isotropic assumption is introduced in section 6 , which allows, together with the new symmetrisation, the decoupling of the scattering matrix and the symmetrised Wigner-Smith matrix, leading eventually to the representation as an exponential functional of the matrix Brownian motion. The relation with the matricial generalization of the Dufresne identity is discussed in section 7 . The representation is used in section 8 in order to derive the first moments for finite length. Finally, in section 9, we discuss the resolvent of the Wigner-Smith matrix in the large $N$ limit, i.e. the Stieltjes transform of the density of eigenvalues.

\section{Wigner-Smith matrix, localisation and exponential functional of the BM}

This section presents some (partly heuristic) arguments explaining the origin of our main result, equation (22), from the localisation properties in multichannel disordered wires. The model under investigation in the article, introduced in section 1.1, is the Schrödinger equation (13) for an $N$ component wave function. We study here the scattering problem, i.e. eigenstates of the form (21). For a given energy $\varepsilon$, we can construct $N$ independent solutions $\left\{\psi^{(a)}(x)\right\}_{a=1, \ldots, N}$, corresponding to inject the incoming wave in one of the $N$ channels. The study of these $N$ solutions can be 'parallelised' if we gather the $N$ independent column vectors in the $N \times N$ matrix wave function

$$
\Psi_{\varepsilon}(x)=\left(\psi^{(1)}(x) \cdots \psi^{(N)}(x)\right)
$$

which behaves, in the disorder free region, as

$$
\Psi_{\varepsilon}(x)=\frac{1}{\sqrt{4 \pi k}}\left(\mathbf{1}_{N} \mathrm{e}^{-\mathrm{i} k(x-L)}+\mathcal{S}(\varepsilon) \mathrm{e}^{\mathrm{i} k(x-L)}\right) \quad \text { for } x \geqslant L .
$$

The solution obeys the Schrödinger equation

$$
-\Psi_{\varepsilon}^{\prime \prime}(x)+V(x) \Psi_{\varepsilon}(x)=\varepsilon \Psi_{\varepsilon}(x) \text { for } x \geqslant 0 .
$$


As shown in appendix A, the wave function matrix is related to the Wigner-Smith matrix by the exact relation

$$
\int_{0}^{L} \mathrm{~d} x \Psi_{\varepsilon}^{\dagger}(x) \Psi_{\varepsilon}(x)=\frac{1}{2 \pi}\left(\mathcal{Q}+\frac{\mathcal{S}-\mathcal{S}^{\dagger}}{4 \mathrm{i} \varepsilon}\right)
$$

which assumes Dirichlet boundary conditions $\Psi_{\varepsilon}(0)=0$.

Equation (27) allows to understand easily the origin of the relation between the Wigner-Smith matrix and exponential functionals of the BM; we follow and extend the argument given in reference [29] for the case $N=1$. In the high energy/weak disorder regime, we can neglect the last term of (27) and write

$$
\mathcal{Q} \simeq 2 \pi \int_{0}^{L} \mathrm{~d} x \Psi_{\varepsilon}^{\dagger}(x) \Psi_{\varepsilon}(x) .
$$

The wave function $\Psi_{\varepsilon}(x)$ presents fast oscillations on the scale $k^{-1}$ while its envelope is a smooth function, damped over scales given by the Lyapunov spectrum.

For $N=1$ (strictly one-dimensional case), we recall the argument of reference [29] leading to the representation (3): the wave function in the disordered region may be parametrised as $\psi(x)=\frac{1}{\sqrt{\pi k}}[\varphi(x) / \varphi(L)] \sin \theta(x)$ where $\varphi(x)$ is an envelope and $\theta(x)$ a phase which controls the rapid oscillations. The presence of $\varphi(L)^{-1}$ ensures the matching on the behaviour (21). In the integral $\int_{0}^{L} \mathrm{~d} x|\psi(x)|^{2}$, one can average over the fast oscillations, which corresponds to perform $\psi(x) \rightarrow \frac{1}{\sqrt{2 \pi k}} \varphi(x) / \varphi(L)$ in the integral. The growth of the envelope is controlled by the Lyapunov exponent $\gamma$, inverse localisation length $\xi=1 / \gamma$ : it is known to obey the SDE $\varphi^{\prime}(x)=[\gamma+\sqrt{\gamma} \eta(x)] \varphi(x)$ [54], where $\eta(x)$ is a normalised Gaussian white noise (the fact that the diffusion and the drift are equal is known as 'single parameter scaling' [55] ; see the recent broader discussion [56]). A change of variable $x \rightarrow L-x$ in the integral eventually leads to the representation (3).

We now extend the argument to the multichannel case. Let us now assume that averaging over the fast oscillations of the matrix wave function corresponds to perform a similar substitution

$$
\Psi_{\varepsilon}(x) \longrightarrow \frac{1}{\sqrt{2 \pi k}} \Phi(x) \Phi(L)^{-1}
$$

in (28), where $\Phi(x)$ describes the smooth evolution of the envelope of the wave function. It is expected to obey the MSDE

$$
\partial_{x} \Phi(x)=(\tilde{\mu} D+\sqrt{D} \eta(x)) \Phi(x)
$$

where $\eta(x)$ a normalised $N \times N$ matrix Gaussian white noise. The drift $\tilde{\mu}$ and the diffusion constant $D$ can be related to the well-known localisation properties from the three following remarks:

- The Lyapunov spectrum of $X^{\prime}(x)=\eta(x) X(x)$ in the orthogonal case has been obtained by Le Jan [57] and Newman [58]: $\gamma_{n}=\frac{\beta}{2}(N-2 n+1)$ for $n \in\{1, \ldots, N\}$ (for the unitary case, see [59]). Thus (30) is related to the Lyapunov spectrum $\gamma_{n}=$ $D[\tilde{\mu}+(\beta / 2)(2 n-1-N)]$ for $n \in\{1, \ldots, N\}$.

- The Lyapunov spectrum characterizing localisation in multichannel disordered wires is known [5] $\gamma_{n} \propto 1+\beta(n-1)$.

- The $N=1$ case coincides with the striclty one dimensional Lyapunov exponent $\gamma_{1}=\frac{\sigma}{8 k^{2}}$ (for high energy) [54]. 
The three remarks lead to $D=\sigma /\left(8 k^{2}\right)$ and $\tilde{\mu}=1+\frac{\beta}{2}(N-1) \equiv \mu$, coincinding with the drift introduced above, equation (19). Thus, the Lyapunov spectrum for $\Phi(x)$ (i.e. for the wave function $\left.\Psi_{\varepsilon}(x)\right)$ is

$$
\gamma_{n}=\frac{\sigma}{8 k^{2}}(1+\beta(n-1)) \quad \text { for } n \in\{1, \ldots, N\}
$$

The localisation length is given by the smallest Lyapunov exponent

$$
\xi=\frac{1}{\gamma_{1}}=\frac{8 k^{2}}{\sigma}
$$

The substitution (29) leads to

$$
\mathcal{Q} \stackrel{\text { conjecture }}{=} \frac{1}{k} \int_{0}^{L} \mathrm{~d} x\left(\Phi(L)^{\dagger}\right)^{-1} \Phi(x)^{\dagger} \Phi(x) \Phi(L)^{-1}
$$

(remind that (29) has not been fully justified). The change of variable $\Lambda(x / \xi)=\Phi(L$ $-x) \Phi(L)^{-1}$, allows to rewrite the functional as

$$
\mathcal{Q} \stackrel{\text { conjecture }}{=} 2 \tau_{\xi} \int_{0}^{L / \xi} \mathrm{d} x \Lambda(x)^{\dagger} \Lambda(x) \quad \text { where } \quad \partial_{x} \Lambda(x)=(-\mu+\eta(x)) \Lambda(x)
$$

for $\Lambda(0)=\mathbf{1}_{N}$. The scale is $2 \tau_{\xi}=\xi / k$. The matrix Dufresne identity states that (34) has a limit law for $L \rightarrow \infty$ : precisely, $\Gamma \stackrel{\text { (law) }}{=}\left(\int_{0}^{\infty} \mathrm{d} x \Lambda(x)^{\dagger} \Lambda(x)\right)^{-1}$ is distributed according to the Wishart distribution

$$
P(\Gamma)=\mathcal{C}_{N, \beta}(\operatorname{det} \Gamma)^{\mu-1-\beta(N-1) / 2} \mathrm{e}^{-(1 / 2) \operatorname{tr}\{\Gamma\}} \text { for } \mu>\frac{\beta}{2}(N-1),
$$

which is proven in section 7 (and for $\beta=1$ in reference [60]). The distribution is defined over the set of positive Hermitian matrices, $\Gamma>0$, i.e. matrices with positive eigenvalues. $\mathcal{C}_{N, \beta}$ is a normalisation constant. Using (19) we recover the distribution (10).

The above derivation makes clear the relation between the statistical properties of the Wigner-Smith matrix and localisation properties, which emphasizes their universal character. However the argumentation of this section has a weakness: the substitution (29) is a rather strong assumption. Adding a unitary matrix $U(x)$, controlled by slow variables, to the wave function would not change the Lyapunov spectrum, however the substitution $\Psi_{\varepsilon}(x) \longrightarrow(2 \pi k)^{-1 / 2} \Phi(x) U(x) U(L)^{-1} \Phi(L)^{-1}$, would not lead to (34). In the next sections, we follow a more rigorous approach based on the analysis of matrix SDE, which generalizes to the mulichannel case the method of reference [31] for $N=1$. We will show that the symmetrised Wigner-Smith matrix admits the representation (34).

\section{Matrix stochastic differential equations for $\mathcal{S}$ and $\mathcal{Q}$}

In this section, we derive the main matrix stochastic differential equation (MSDE) for the scattering matrix and the Wigner-Smith matrix, at the heart of our analysis. A convenient starting point is to introduce the Riccati matrix

$$
Z(x)=\Psi^{\prime}(x) \Psi(x)^{-1}
$$

(we drop the energy label ${ }_{\varepsilon}$ in the wave function). From (26), it is straightforward to get

$$
\partial_{x} Z(x)=-\varepsilon \mathbf{1}_{N}-Z(x)^{2}+V(x) \quad \text { with } \varepsilon=k^{2},
$$


with the initial condition $Z(0)=\infty \mathbf{1}_{N}$, corresponding to the Dirichlet condition $\Psi(0)=0 \mathbf{1}_{N}$. Equation (25) makes clear that the scattering matrix can be expressed as

$$
\mathcal{S}=\left[k \mathbf{1}_{N}-\mathrm{i} Z(L)\right]\left[k \mathbf{1}_{N}+\mathrm{i} Z(L)\right]^{-1},
$$

or equivalently

$$
Z(L)=\mathrm{i} k\left(\mathcal{S}-\mathbf{1}_{N}\right)\left(\mathcal{S}+\mathbf{1}_{N}\right)^{-1}
$$

Using (37), we can write an equation describing the evolution of $\mathcal{S}$ upon increasing $L$ :

$$
\partial_{L} \mathcal{S}=2 \mathrm{i} k \mathcal{S}+\frac{1}{2 \mathrm{i} k}\left(\mathbf{1}_{N}+\mathcal{S}\right) V(L)\left(\mathbf{1}_{N}+\mathcal{S}\right)
$$

One can check that this equation preserves the unitarity $\mathcal{S}^{\dagger}=\mathcal{S}^{-1}$. Additionally, for $\beta=1$, we have $V(x)^{\mathrm{T}}=V(x)$ therefore $\mathcal{S}^{\mathrm{T}}=\mathcal{S}$.

Derivation of (40) with respect to $\varepsilon=k^{2}$ provides the MSDE satisfied by $\mathcal{Q}$ :

$$
\partial_{L} \mathcal{Q}=\frac{\mathbf{1}_{N}}{k}+\frac{1}{2 \mathrm{i} k}\left[\mathcal{Q} V(L)\left(\mathbf{1}_{N}+\mathcal{S}\right)-\left(\mathbf{1}_{N}+\mathcal{S}^{\dagger}\right) V(L) \mathcal{Q}\right]+\frac{1}{4 k^{3}}\left(\mathbf{1}_{N}+\mathcal{S}^{\dagger}\right) V(L)\left(\mathbf{1}_{N}+\mathcal{S}\right)
$$

In the next section, we analyse these equations in the weak disorder limit and identify fast and slow variables. Elimination of fast variables leads to simplified MSDE describing the variables on large scales.

\section{Averaging over fast variables in the weak disorder limit}

In the weak disorder limit $\sigma \ll k^{3}$, the evolution of $\mathcal{S}$ and $\mathcal{Q}$ is controlled by two length scales:

- The wavelength $\lambda=1 / k$ controls the fast oscillations (which are present in the absence of disorder, $V(x)=0)$;

- The localisation length $\xi=8 k^{2} / \sigma \gg \lambda$, or the mean free path $\ell_{\mathrm{e}} \sim \xi / N$, which is the typical length scale for the evolution of the other variables.

The idea is to perform some averaging over short scale $\lambda=1 / k$ to get rid of the fast oscillations and obtain equations describing the evolution of $\mathcal{S}$ and $\mathcal{Q}$ on the larger scale $\xi \sim\left(k^{3} / \sigma\right) \lambda$. The main difficulty is that MSDE, as equation (40), must be manipulated with care. A rigorous approach is to relate the MSDE to a Fokker-Planck equation for a matrix distribution: this can be achieved for matrix random process [59], however it is quite cumbersome. In the present paper, we discuss this approach in appendix B for the specific case $N=2$ and $\beta=1$. Here we have found more convenient to work directly with MSDE by identifying effective independent noises. We have kept some control over the method by comparing the outcome with the more rigorous Fokker-Planck approach in a specific case (appendix B).

\subsection{The scattering matrix}

The starting point is to remove the fast oscillations by introducing

$$
\widetilde{\mathcal{S}}=\mathrm{e}^{-2 \mathrm{i} k x} \mathcal{S}
$$


(from now on, $x$ must be understood as the size of the disordered region). From equation (40), we obtain the MSDE satisfied by $\widetilde{\mathcal{S}}$ :

$$
\partial_{x} \widetilde{\mathcal{S}}=\frac{1}{2 \mathrm{i} k}\left(\mathrm{e}^{-\mathrm{i} k x}+\widetilde{\mathcal{S}} \mathrm{e}^{\mathrm{i} k x}\right) V(x)\left(\mathrm{e}^{-\mathrm{i} k x}+\mathrm{e}^{\mathrm{i} k x} \widetilde{\mathcal{S}}\right) .
$$

Thus

$$
\begin{aligned}
\partial_{x} \widetilde{\mathcal{S}}= & \frac{1}{2 \mathrm{i} k}\left[\left(\mathbf{1}_{N}+\widetilde{\mathcal{S}}\right) \cos k x-\mathrm{i}\left(\mathbf{1}_{N}-\widetilde{\mathcal{S}}\right) \sin k x\right] V(x)\left[\cos k x\left(\mathbf{1}_{N}+\widetilde{\mathcal{S}}\right)\right. \\
& \left.-\mathrm{i} \sin k x\left(\mathbf{1}_{N}-\widetilde{\mathcal{S}}\right)\right] .
\end{aligned}
$$

We can rewrite this equation as

$$
\partial_{x} \widetilde{\mathcal{S}}=\frac{1}{2 \mathrm{i} k}\left\{\left[V_{1}(x)-\mathrm{i} V_{2}(x)\right]+\widetilde{\mathcal{S}}\left[V_{1}(x)+\mathrm{i} V_{2}(x)\right] \widetilde{\mathcal{S}}+\widetilde{\mathcal{S}} V(x)+V(x) \widetilde{\mathcal{S}}\right\},
$$

where we have introduced

$$
V_{1}(x)=\cos (2 k x) V(x) \quad \text { and } \quad V_{2}(x)=\sin (2 k x) V(x) .
$$

In the high energy limit, the trigonometric functions oscillate fast compared to the typical length scale for the evolution of $\widetilde{\mathcal{S}}$. In this limit, $V_{1}(x), V_{2}(x)$ and $V(x)$ become independent Gaussian white noises, as we now demonstrate. Let us compute the correlations between the different processes:

$$
\begin{aligned}
\left\langle\int_{0}^{x}\left(V_{1}\right)_{a b} \int_{0}^{x^{\prime}}\left(V_{1}\right)_{c d}^{*}\right\rangle & =\sigma \int_{0}^{\min \left(x, x^{\prime}\right)} C_{a b, c d} \cos ^{2}(2 k t) \mathrm{d} t \simeq \frac{\sigma}{2} C_{a b, c d} \min \left(x, x^{\prime}\right), \\
\left\langle\int_{0}^{x}\left(V_{1}\right)_{a b} \int_{0}^{x^{\prime}}\left(V_{2}\right)_{c d}^{*}\right\rangle & =\sigma \int_{0}^{\min \left(x, x^{\prime}\right)} C_{a b, c d} \cos (2 k t) \sin (2 k t) \mathrm{d} t \simeq 0 \\
\left\langle\int_{0}^{x}\left(V_{1}\right)_{a b} \int_{0}^{x^{\prime}} V_{c d}^{*}\right\rangle & =\sigma \int_{0}^{\min \left(x, x^{\prime}\right)} C_{a b, c d} \cos (2 k t) \mathrm{d} t \simeq 0 .
\end{aligned}
$$

The same properties holds for $V_{2}$. Over large scales $\gg \lambda=1 / k$, the correlations between the three noises $V_{1}(x), V_{2}(x)$ and $V(x)$ vanish. Due to their Gaussian nature, they can thus be considered as three independent matricial Gaussian white noises, such that

$$
V_{1}(x) \stackrel{(\text { law })}{=} V_{2}(x) \stackrel{(\text { law })}{=} \frac{1}{\sqrt{2}} V(x) .
$$

Remark: from our derivation of appendix B, equation (45) must be interpreted in the Stratonovich sense.

\subsection{The Wigner-Smith matrix}

We introduce $\mathcal{S}=\mathrm{e}^{2 \mathrm{i} k x} \widetilde{\mathcal{S}}$ in the $\operatorname{MSDE}(41)$ :

$$
\begin{aligned}
\partial_{x} \mathcal{Q}= & \frac{\mathbf{1}_{N}}{k}+\frac{1}{2 \mathrm{i} k}\left\{\mathcal{Q}\left[V+V_{1} \widetilde{\mathcal{S}}+\mathrm{i} V_{2} \widetilde{\mathcal{S}}\right]-\left[V+\widetilde{\mathcal{S}}^{\dagger} V_{1}-\mathrm{i} \widetilde{\mathcal{S}}^{\dagger} V_{2}\right] \mathcal{Q}\right\} \\
& +\frac{1}{4 k^{3}}\left[V+\widetilde{\mathcal{S}}^{\dagger} V \widetilde{\mathcal{S}}+\widetilde{\mathcal{S}}^{\dagger}\left(V_{1}-\mathrm{i} V_{2}\right)+\left(V_{1}+\mathrm{i} V_{2}\right) \widetilde{\mathcal{S}}\right]
\end{aligned}
$$


In the high energy/weak disorder limit, we can drop the last term of (51) which is subleading (anticipating on the result, $\mathcal{Q}$ typically grows exponentially with the system size, while the neglected term is bounded). We obtain

$$
\partial_{x} \mathcal{Q} \simeq \frac{\mathbf{1}_{N}}{k}+\frac{1}{2 \mathrm{i} k}\left\{\mathcal{Q}\left[V+V_{1} \widetilde{\mathcal{S}}+\mathrm{i} V_{2} \widetilde{\mathcal{S}}\right]-\left[V+\widetilde{\mathcal{S}}^{\dagger} V_{1}-\mathrm{i} \widetilde{\mathcal{S}}^{\dagger} V_{2}\right] \mathcal{Q}\right\}
$$

We recall that $\widetilde{\mathcal{S}}$ satisfies (45). Both (45) and (52) must be interpreted in the Stratonovich sense.

\section{The 'square-root trick' and a new symmetrisation of the Wigner-Smith matrix}

In chaotic cavities, an important step for the determination of the distribution of the Wigner-Smith matrix eigenvalues was the introduction of the symmetrised Wigner-Smith matrix $\mathcal{Q}_{s}=\mathcal{S}^{1 / 2} \mathcal{Q} \mathcal{S}^{-1 / 2}$ [18]. This makes $\mathcal{S}$ and $\mathcal{Q}_{s}$ independent and ensures that $\mathcal{Q}_{s}$ is real symmetric for $\beta=1$. However, such a symmetrisation is not possible for multichannel 1D wires as we cannot get a simple MSDE satisfied by $\mathcal{S}^{1 / 2}$. To circumvent this problem we follow here a different strategy: we introduce two unitary matrices $\mathcal{U}_{L}$ and $\mathcal{U}_{R}$ which satisfy the equations

$$
\begin{aligned}
& \partial_{x} \mathcal{U}_{L}=\frac{1}{2 \mathrm{i} k}\left(\frac{1}{2}\left(V_{1}-\mathrm{i} V_{2}\right) \mathcal{U}_{R}^{-1}+\frac{1}{2} \mathcal{U}_{L} \mathcal{U}_{R}\left(V_{1}+\mathrm{i} V_{2}\right) \mathcal{U}_{L}+V \mathcal{U}_{L}\right) \\
& \partial_{x} \mathcal{U}_{R}=\frac{1}{2 \mathrm{i} k}\left(\frac{1}{2} \mathcal{U}_{L}^{-1}\left(V_{1}-\mathrm{i} V_{2}\right)+\frac{1}{2} \mathcal{U}_{R}\left(V_{1}+\mathrm{i} V_{2}\right) \mathcal{U}_{L} \mathcal{U}_{R}+\mathcal{U}_{R} V\right)
\end{aligned}
$$

One can easily check that these equations preserve the unitarity of both $\mathcal{U}_{L}$ and $\mathcal{U}_{R}$. Furthermore, we can deduce from (53) and (54) an SDE for the matrix $\mathcal{U}_{L} \mathcal{U}_{R}$, which coincides with equation (45), thus

$$
\widetilde{\mathcal{S}}=\mathcal{U}_{L} \mathcal{U}_{R}
$$

This provides a factorisation of the scattering matrix which can be used to take some sort of 'square root' (a similar trick was used in reference [26] in the orthogonal case). Furthermore, for orthogonal symmetry class, we can easily check that $\mathcal{U}_{R}=\mathcal{U}_{L}^{\mathrm{T}}$, thus

$$
\widetilde{\mathcal{S}}=\mathcal{U}_{L} \mathcal{U}_{L}^{\mathrm{T}}=\mathcal{U}_{R}^{\mathrm{T}} \mathcal{U}_{R}=\widetilde{\mathcal{S}}^{\mathrm{T}} \quad \text { for } \beta=1
$$

This allows us to introduce an alternative symmetrisation of the Wigner-Smith matrix

$$
\widetilde{\mathcal{Q}}=\mathcal{U}_{R} \mathcal{Q} \mathcal{U}_{R}^{\dagger}=\mathrm{e}^{-2 \mathrm{i} k x} \mathcal{U}_{L}^{\dagger} \partial_{\varepsilon}\left(\mathrm{e}^{2 \mathrm{i} k x} \mathcal{U}_{L} \mathcal{U}_{R}\right) \mathcal{U}_{R}^{\dagger},
$$

where we have used that $\mathcal{S}=\mathrm{e}^{2 \mathrm{i} k x} \widetilde{\mathcal{S}}=\mathrm{e}^{2 \mathrm{i} k x} \mathcal{U}_{L} \mathcal{U}_{R}$.

We can obtain the MSDE satisfied by $\widetilde{\mathcal{Q}}$ by combining equations (52) and (54). We thus obtain

$$
\partial_{x} \widetilde{\mathcal{Q}}=\frac{\mathbf{1}_{N}}{k}+\frac{1}{2 k}\left(\widetilde{\mathcal{Q}} W\left[\mathcal{U}_{L}, \mathcal{U}_{R}, V_{1}, V_{2}\right]+W\left[\mathcal{U}_{L}, \mathcal{U}_{R}, V_{1}, V_{2}\right] \widetilde{\mathcal{Q}}\right)
$$

where we have introduced the Hermitian matrix

$$
W\left[\mathcal{U}_{L}, \mathcal{U}_{R}, V_{1}, V_{2}\right]=\frac{1}{2 \mathrm{i}}\left(\mathcal{U}_{R} V_{1} \mathcal{U}_{L}-\mathcal{U}_{L}^{\dagger} V_{1} \mathcal{U}_{R}^{\dagger}\right)+\frac{1}{2}\left(\mathcal{U}_{R} V_{2} \mathcal{U}_{L}+\mathcal{U}_{L}^{\dagger} V_{2} \mathcal{U}_{R}^{\dagger}\right)
$$


Integrating (58) over $[0, L]$ leads to

$$
\widetilde{\mathcal{Q}}=\frac{1}{k} X(L)\left(\int_{0}^{L} \mathrm{~d} x X(x)^{-1} X^{\dagger}(x)^{-1}\right) X^{\dagger}(L),
$$

where $X(x)$ solves the MSDE

$$
\partial_{x} X(x)=\frac{1}{2 k} W\left[\mathcal{U}_{L}, \mathcal{U}_{R}, V_{1}, V_{2}\right] X(x) .
$$

The problem is now to study the equation (61), with $\mathcal{U}_{L}$ and $\mathcal{U}_{R}$ satisfying equations (53) and (54), respectively.

We stress that, up to now, we have made no assumption on the distribution of the random potential $V$ (and $V_{1} \stackrel{\text { (law) }}{=} V_{2} \stackrel{\text { (law) }}{=} V / \sqrt{2}$ ), like isotropy. Gaussian distribution was assumed in order to simplify the discussion, although it is not essential as the analysis only involves the second moment of the disorder: see equations (47)-(49).

\section{Isotropic case: decoupling of $\widetilde{\mathcal{S}}$ and $\widetilde{\mathcal{Q}}$}

We now rescale the matrix Gaussian white noise as $V \rightarrow \sqrt{\sigma} \eta$, with $\left\langle\eta_{a b}(x) \eta_{c d}^{*}\left(x^{\prime}\right)\right\rangle=$ $C_{a b, c d} \delta\left(x-x^{\prime}\right)$. In this section, we use the mathematical notation for SDE, based on $\mathrm{d} B(x)=$ $\eta(x) \mathrm{d} x$ satisfying

$$
\mathrm{d} B_{a b}(x) \mathrm{d} B_{c d}^{*}(x)=C_{a b, c d} \mathrm{~d} x \quad \text { with } C_{a b, c d}=\frac{\beta}{2} \delta_{a c} \delta_{b d}+\left(1-\frac{\beta}{2}\right) \delta_{a d} \delta_{b c}
$$

We deduce the useful relation

$$
\mathrm{d} B(x) \mathcal{O} \mathrm{d} B(x)=\left[\frac{\beta}{2} \operatorname{tr}\{\mathcal{O}\} \mathbf{1}_{N}+\left(1-\frac{\beta}{2}\right) \mathcal{O}^{\mathrm{T}}\right] \mathrm{d} x
$$

for any matrix $\mathcal{O}$ uncorrelated with $\mathrm{d} B(x)$. In particular, setting $\mathcal{O}=\mathbf{1}_{N}$, we get

$$
\mathrm{d} B(x)^{2}=\mu \mathrm{d} x \mathbf{1}_{N} \quad \text { where } \mu=1+\frac{\beta}{2}(N-1) .
$$

\subsection{Warm up: case $N=1$}

It is helpful to start the analysis by considering the case $N=1$ : averaging over the fast variable was performed in the Fokker-Planck equation in reference [31] (see also [30]). Let us see how equations (52) and (45) yield the known result (3) by manipulating the SDE. Let us denote $\widetilde{\mathcal{S}}=\mathrm{e}^{\mathrm{i} \alpha}$. Equations (45) and (52) reduce to

$$
\begin{aligned}
& \mathrm{d} \alpha(x)=-\frac{\sqrt{\sigma}}{k}\left[\mathrm{~d} B(x)+\frac{1}{\sqrt{2}}\left(\cos \alpha \mathrm{d} B_{1}(x)-\sin \alpha \mathrm{d} B_{2}(x)\right)\right], \\
& \mathrm{d} \mathcal{Q}(x)=\frac{1}{k}\left[\mathrm{~d} x+\frac{\sqrt{\sigma}}{\sqrt{2}}\left(\sin \alpha \mathrm{d} B_{1}(x)+\cos \alpha \mathrm{d} B_{2}(x)\right) \mathcal{Q}\right],
\end{aligned}
$$

where $B(x), B_{1}(x)$ and $B_{2}(x)$ are three independent normalised Brownian motions. As mentioned above, these two equations are interpreted in the Stratonovich sense. Relating them to 
SDE in the Itô sense, we get here the same equations. Let us now choose the Itô convention for convenience. We define two new noises

$$
\begin{aligned}
& \mathrm{d} w_{1}(x)=\cos \alpha(x) \mathrm{d} B_{1}(x)-\sin \alpha(x) \mathrm{d} B_{2}(x) \\
& \mathrm{d} w_{2}(x)=\sin \alpha(x) \mathrm{d} B_{1}(x)+\cos \alpha(x) \mathrm{d} B_{2}(x) .
\end{aligned}
$$

Since we work with the Itô convention, we have $\left\langle\mathrm{d} w_{1}(x)\right\rangle=\left\langle\mathrm{d} w_{2}(x)\right\rangle=0$. The strength of the noises is $\mathrm{d} w_{1}(x)^{2}=\mathrm{d} w_{2}(x)^{2}=\mathrm{d} x$ and they are clearly uncorrelated, $\mathrm{d} w_{1}(x) \mathrm{d} w_{2}(x)=0$. The two new noises are thus independent, and we can rewrite

$$
\begin{aligned}
& \mathrm{d} \alpha(x)=-\frac{\sqrt{\sigma}}{k}\left[\mathrm{~d} B(x)+\frac{1}{\sqrt{2}} \mathrm{~d} w_{1}(x)\right] \\
& \mathrm{d} \mathcal{Q}(x)=\frac{1}{k}\left[\mathrm{~d} x+\frac{\sqrt{\sigma}}{\sqrt{2}} \mathrm{~d} w_{2}(x) \mathcal{Q}\right]
\end{aligned}
$$

All the manipulations have assumed that SDE are in the Itô sense. Converting the second equation to Stratonovich convention, we obtain

$$
\partial_{x} \mathcal{Q} \stackrel{\text { (law) }}{=} \frac{1}{k}+\left(\frac{V(x)}{\sqrt{2} k}-\frac{\sigma}{4 k^{2}}\right) \mathcal{Q}=\frac{1}{k}+\left(\frac{2 \eta(x)}{\sqrt{\xi}}-\frac{2}{\xi}\right) \mathcal{Q},
$$

where $V(x)$ is the original potential and $\eta(x)$ a normalised Gaussian white noise. Thus, we have recovered the result of reference [31] and equation (3), following a more simple procedure.

\subsection{Strategy for $N>1$}

Let us now consider the case of isotropic noise, which corresponds to a correlator of the form (14) and (15). We consider equation (61) instead of the symmetrised Wigner-Smith matrix $\widetilde{\mathcal{Q}}$, since they can be easily related via (60).

Let us first rewrite equations (53), (54) and (61) in the form

$$
\begin{aligned}
\mathrm{d} X & =\frac{\sqrt{\sigma / 2}}{2 k} W\left[\mathcal{U}_{L}, \mathcal{U}_{R}, \mathrm{~d} B_{1}, \mathrm{~d} B_{2}\right] X \\
\mathrm{~d} \mathcal{U}_{L} & =\frac{\sqrt{\sigma}}{2 \mathrm{i} k}\left(\frac{1}{\sqrt{2}} \mathcal{U}_{L} W_{u}\left[\mathcal{U}_{L}, \mathcal{U}_{R}, \mathrm{~d} B_{1}, \mathrm{~d} B_{2}\right]+\mathrm{d} B(x) \mathcal{U}_{L}\right), \\
\mathrm{d} \mathcal{U}_{R} & =\frac{\sqrt{\sigma}}{2 \mathrm{i} k}\left(\frac{1}{\sqrt{2}} W_{u}\left[\mathcal{U}_{L}, \mathcal{U}_{R}, \mathrm{~d} B_{1}, \mathrm{~d} B_{2}\right] \mathcal{U}_{R}+\mathcal{U}_{R} \mathrm{~d} B(x)\right),
\end{aligned}
$$

where $W$ is given by equation (59) and we have denoted

$$
W_{u}\left[\mathcal{U}_{L}, \mathcal{U}_{R}, \mathrm{~d} B_{1}, \mathrm{~d} B_{2}\right]=\frac{1}{2}\left(\mathcal{U}_{L}^{\dagger} \mathrm{d} B_{1} \mathcal{U}_{R}^{\dagger}+\mathcal{U}_{R} \mathrm{~d} B_{1} \mathcal{U}_{L}\right)+\frac{1}{2 \mathrm{i}}\left(\mathcal{U}_{L}^{\dagger} \mathrm{d} B_{2} \mathcal{U}_{R}^{\dagger}-\mathcal{U}_{R} \mathrm{~d} B_{2} \mathcal{U}_{L}\right)
$$

$B_{1}, B_{2}$ and $B$ are now three independent normalised Brownian motions, each satisfying (62). The idea is the following: since the $B_{i}$ 's are isotropic, the noises $W$ and $W_{u}$ can be shown to be independent, and we can thus decouple the equations for $\mathcal{U}_{L}$ and $\mathcal{U}_{R}$ from the equation for $X$ (and thus $\widetilde{\mathcal{Q}}$ ).

In order to do so, the procedure is the following:

(a) Convert the stochastic equations from Stratonovich to Itô convention in order to decouple the matrices from the noises at coinciding points; 
(b) Show that the two noises $W$ and $W_{u}$ are independent Gaussian white noises (independently of $\mathcal{U}_{L}$ and $\left.\mathcal{U}_{R}\right)$, and then replace them with new ones with the same distribution, but which do no involve $\mathcal{U}_{L}$ or $\mathcal{U}_{R}$

(c) Convert the new equations back to Stratonovich convention.

Concerning the first point, we only need to convert the equation for $X$, since we will no longer be interested in the unitary matrices.

\subsection{Conversion to the Itô convention}

Converting a stratonovich MSDE (72) into the Itô convention brings an additional drift ${ }^{8}$

$$
\mathfrak{D} \mathbf{r i f t}=\frac{1}{2} \sum_{i, j}\left((\mathrm{~d} X)_{i j} \frac{\partial(\mathrm{d} X)}{\partial X_{i j}}+\left(\mathrm{d} \mathcal{U}_{L}\right)_{i j} \frac{\partial(\mathrm{d} X)}{\partial\left(\mathcal{U}_{L}\right)_{i j}}+\left(\mathrm{d} \mathcal{U}_{R}\right)_{i j} \frac{\partial(\mathrm{d} X)}{\partial\left(\mathcal{U}_{R}\right)_{i j}}\right)
$$

The MSDE $\mathrm{d} X(72)$ depends on $\mathcal{U}_{L}$ and $\mathcal{U}_{R}$ only through $W$, which is linear in $\mathcal{U}_{L}, \mathcal{U}_{R}, \mathcal{U}_{L}^{\dagger}$ and $\mathcal{U}_{R}^{\dagger}$. Thus,

$$
\begin{aligned}
\mathfrak{D r i f t}= & \frac{\sqrt{\sigma / 2}}{4 k}\left(W\left[\mathcal{U}_{L}, \mathcal{U}_{R}, \mathrm{~d} B_{1}, \mathrm{~d} B_{2}\right] \mathrm{d} X+W\left[\mathrm{~d} \mathcal{U}_{L}, \mathcal{U}_{R}, \mathrm{~d} B_{1}, \mathrm{~d} B_{2}\right] X+W\left[\mathcal{U}_{L}, \mathrm{~d} \mathcal{U}_{R}, \mathrm{~d} B_{1}, \mathrm{~d} B_{2}\right] X\right) \\
= & \frac{\sqrt{\sigma / 2}}{4 k}\left(\frac{\sqrt{\sigma / 2}}{2 k} W\left[\mathcal{U}_{L}, \mathcal{U}_{R}, \mathrm{~d} B_{1}, \mathrm{~d} B_{2}\right]^{2}+W\left[\mathrm{~d} \mathcal{U}_{L}, \mathcal{U}_{R}, \mathrm{~d} B_{1}, \mathrm{~d} B_{2}\right]\right. \\
& \left.+W\left[\mathcal{U}_{L}, \mathrm{~d} \mathcal{U}_{R}, \mathrm{~d} B_{1}, \mathrm{~d} B_{2}\right]\right) X
\end{aligned}
$$

Let us look at the second term. When replacing $W$ and $\mathrm{d} \mathcal{U}_{L}$ by their expressions, we obtain products of the different noises $\mathrm{d} B, \mathrm{~d} B_{1}$ and $\mathrm{d} B_{2}$. Since they are independent, the only non vanishing terms will involve products of the same noise. We thus obtain

$$
\begin{aligned}
W\left[\mathrm{~d} \mathcal{U}_{L}, \mathcal{U}_{R}, \mathrm{~d} B_{1}, \mathrm{~d} B_{2}\right]= & -\frac{\sqrt{\sigma / 2}}{4 k}\left(\mathcal{U}_{R} \mathrm{~d} B_{1}^{2} \mathcal{U}_{R}^{\dagger}+\mathcal{U}_{R} \mathrm{~d} B_{1} \mathcal{U}_{L} \mathcal{U}_{R} \mathrm{~d} B_{1} \mathcal{U}_{L}\right. \\
& \left.+\mathcal{U}_{R} \mathrm{~d} B_{2}^{2} \mathcal{U}_{R}^{\dagger}-\mathcal{U}_{R} \mathrm{~d} B_{2} \mathcal{U}_{L} \mathcal{U}_{R} \mathrm{~d} B_{2} \mathcal{U}_{L}+\text { h.c. }\right)
\end{aligned}
$$

Since $\mathrm{d} B_{1}(x) \stackrel{(\text { law })}{=} \mathrm{d} B_{2}(x) \stackrel{(\text { law })}{=} \mathrm{d} B(x)$, this reduces to

$$
W\left[\mathrm{~d} \mathcal{U}_{L}, \mathcal{U}_{R}, V_{1}, V_{2}\right]=-\frac{\sqrt{\sigma / 2}}{2 k} \mathcal{U}_{R} \mathrm{~d} B^{2} \mathcal{U}_{R}^{\dagger}
$$

Similarly,

$$
W\left[\mathcal{U}_{L}, \mathrm{~d} \mathcal{U}_{R}, V_{1}, V_{2}\right]=-\frac{\sqrt{\sigma / 2}}{2 k} \mathcal{U}_{L}^{\dagger} \mathrm{d} B^{2} \mathcal{U}_{L}
$$

\footnotetext{
${ }^{8}$ The simplest way to perform the Stratonovich $\rightarrow$ Itô conversion is as follows. Consider the Stratonovich SDE $\mathrm{d} x(t)=\alpha(x(t)) \mathrm{d} t+b(x(t)) \mathrm{d} W(t)$, and define $(\mathrm{d} x)_{\text {noise }}=b(x) \mathrm{d} W(t)$. The corresponding Itô equation is obtained by writing $\mathrm{d} x(t)=\alpha(x) \mathrm{d} t+b\left(x+\frac{1}{2}(\mathrm{~d} x)_{\text {noise }}\right) \mathrm{d} W(t)=a(x) \mathrm{d} t+b(x) \mathrm{d} W(t)$ with $a(x)=\alpha(x)+\frac{1}{2} b^{\prime}(x) b(x)$, where we have used $\mathrm{d} W(t)^{2}=\mathrm{d} t$. This simple procedure can be applied whatever the nature of the process is (scalar, vector, matrix, ....).
} 
So that we finally get

$$
\mathfrak{D r i f t}=\frac{\sigma}{16 k^{2}} W\left[\mathcal{U}_{L}, \mathcal{U}_{R}, \mathrm{~d} B_{1}, \mathrm{~d} B_{2}\right]^{2} X-\frac{\sigma}{16 k^{2}}\left(\mathcal{U}_{R} \mathrm{~d} B^{2} \mathcal{U}_{R}^{\dagger}+\mathcal{U}_{L}^{\dagger} \mathrm{d} B^{2} \mathcal{U}_{L}\right) X .
$$

This equation holds for any type of correlated matrix noise $\mathrm{d} B_{1}(x)$. Now making the assumption that the noise is isotropic, we deduce from (64),

$$
\text { Drift }=\frac{\sigma}{16 k^{2}} W\left[\mathcal{U}_{L}, \mathcal{U}_{R}, \mathrm{~d} B_{1}, \mathrm{~d} B_{2}\right]^{2} X-\frac{\sigma}{8 k^{2}}\left(1+\beta \frac{N-1}{2}\right) X \mathrm{~d} x .
$$

We do not evaluate the first term now, as it will cancel out in the following when we will get back to the Stratonovich form. Nevertheless, it can be easily evaluated from the correlator of $W$, which we now analyse.

\subsection{Characterisation of the effective noises $W$ and $W_{u}$}

Let us now study the distribution of the noises $W\left[\mathcal{U}_{L}, \mathcal{U}_{R}, \mathrm{~d} B_{1}, \mathrm{~d} B_{2}\right]$ and $W_{u}\left[\mathcal{U}_{L}, \mathcal{U}_{R}, \mathrm{~d} B_{1}, \mathrm{~d} B_{2}\right]$, given respectively by equations (59) and (75). We first compute

$$
\begin{aligned}
W_{a b} W_{c d}^{*}= & W_{a b} W_{d c}=-\frac{1}{4}\left(\mathcal{U}_{R} \mathrm{~d} B_{1} \mathcal{U}_{L}-\mathcal{U}_{L}^{\dagger} \mathrm{d} B_{1} \mathcal{U}_{R}^{\dagger}\right)_{a b}\left(\mathcal{U}_{R} \mathrm{~d} B_{1} \mathcal{U}_{L}-\mathcal{U}_{L}^{\dagger} \mathrm{d} B_{1} \mathcal{U}_{R}^{\dagger}\right)_{d c} \\
& +\frac{1}{4}\left(\mathcal{U}_{R} \mathrm{~d} B_{2} \mathcal{U}_{L}+\mathcal{U}_{L}^{\dagger} \mathrm{d} B_{2} \mathcal{U}_{R}^{\dagger}\right)_{a b}\left(\mathcal{U}_{R} \mathrm{~d} B_{2} \mathcal{U}_{L}+\mathcal{U}_{L}^{\dagger} \mathrm{d} B_{2} \mathcal{U}_{R}^{\dagger}\right)_{d c}
\end{aligned}
$$

Expanding and keeping only the non-vanishing terms, and using that $\mathrm{d} B_{1} \stackrel{\text { (law) }}{=} \mathrm{d} B_{2} \stackrel{\text { (law) }}{=} \mathrm{d} B$, we obtain

$$
\begin{aligned}
W_{a b} W_{c d}^{*}= & \frac{1}{2} \sum_{p q r s}\left(\left(\mathcal{U}_{R}\right)_{a p}\left(\mathcal{U}_{L}\right)_{q b}\left(\mathcal{U}_{L}^{\dagger}\right)_{d r}\left(\mathcal{U}_{R}^{\dagger}\right)_{s c}+\left(\mathcal{U}_{L}^{\dagger}\right)_{a p}\left(\mathcal{U}_{R}^{\dagger}\right)_{q b}\left(\mathcal{U}_{R}\right)_{d r}\left(\mathcal{U}_{L}\right)_{s c}\right) \\
& \times \mathrm{d} B_{p q}(x) \mathrm{d} B_{s r}(x)^{*}
\end{aligned}
$$

In the isotropic case, using the expression of the correlator (62), we get

$$
W_{a b}\left[\mathcal{U}_{L}, \mathcal{U}_{R}, \mathrm{~d} B_{1}, \mathrm{~d} B_{2}\right] W_{c d}^{*}\left[\mathcal{U}_{L}, \mathcal{U}_{R}, \mathrm{~d} B_{1}, \mathrm{~d} B_{2}\right]=C_{a b, c d} \mathrm{~d} x
$$

Similarly, we obtain

$$
\begin{aligned}
W_{a b}\left(W_{u}\right)_{c d}^{*}= & \frac{1}{4 \mathrm{i}} \sum_{p q r s}\left(\left(\mathcal{U}_{R}\right)_{a p}\left(\mathcal{U}_{L}\right)_{q b}\left(\mathcal{U}_{L}^{\dagger}\right)_{d r}\left(\mathcal{U}_{R}^{\dagger}\right)_{s c}-\left(\mathcal{U}_{L}^{\dagger}\right)_{a p}\left(\mathcal{U}_{R}^{\dagger}\right)_{q b}\left(\mathcal{U}_{R}\right)_{d r}\left(\mathcal{U}_{L}\right)_{s c}\right) \\
& \times \mathrm{d} B_{1}(x)_{p q} \mathrm{~d} B_{1}(x)_{s r}^{*}
\end{aligned}
$$

which, in the isotropic case yields

$$
W_{a b}\left[\mathcal{U}_{L}, \mathcal{U}_{R}, \mathrm{~d} B_{1}, \mathrm{~d} B_{2}\right]\left(W_{u}^{*}\right)_{c d}\left[\mathcal{U}_{L}, \mathcal{U}_{R}, \mathrm{~d} B_{1}, \mathrm{~d} B_{2}\right]=0
$$

showing that $W$ and $W_{u}$ are uncorrelated. Therefore, we have shown that

$$
W\left[\mathcal{U}_{L}, \mathcal{U}_{R}, \mathrm{~d} B_{1}(x), \mathrm{d} B_{2}(x)\right] \stackrel{(\text { law })}{=} \mathrm{d} B(x),
$$

so that we can rewrite the MSDE (72) as

$$
\mathrm{d} X=(\mathfrak{D r i f t}) X+\frac{\sqrt{\sigma / 2}}{2 k} \mathrm{~d} B(x) X \quad(\text { Itô) },
$$


where the drift is given by equation (82). Isotropy has been used to perform unitary transformations such that the unitary matrices $\mathcal{U}_{L}$ and $\mathcal{U}_{R}$ can be removed from the MSDE for $X(x)$. Eventually, we have obtained an MSDE which involves no other matrix than $X$.

\subsection{Back to the Stratonovich convention}

We can now convert the Itô equation (89) into a Stratonovich one. One has to add the drift term

$$
-\frac{1}{2} \sum_{i j}(\mathrm{~d} X)_{i j} \frac{\partial(\mathrm{d} X)}{\partial X_{i j}}=-\frac{\sigma}{16 k^{2}} \mathrm{~d} B(x)^{2} X
$$

to the Itô equation. This cancels out the first term in (82), and we thus get

$$
\partial_{x} X=-\frac{\mu}{\xi} X+\frac{1}{\sqrt{\xi}} \eta(x) X \quad(\text { Stratonovich })
$$

where we recall that $\mu=1+\beta \frac{N-1}{2}$ and $\mathrm{d} B(x)=\eta(x) \mathrm{d} x$, so that $\eta(x)$ is a Hermitian Gaussian white noise. Therefore, the matrix $X$ is an exponential of a matrix Brownian motion, equation (23). The symmetrised Wigner-Smith matrix $\widetilde{\mathcal{Q}}$ is expressed as a functional of this exponential of Brownian motion via equation (60). This extends the result known for $N=1$ to higher number of channels.

From the expression (60) of $\widetilde{\mathcal{Q}}$ and the stochastic equation for $X$, equation (91), we can derive the MSDE

$$
\frac{\partial}{\partial L} \widetilde{\mathcal{Q}}=\frac{\mathbf{1}_{N}}{k}-\frac{2 \mu}{\xi} \widetilde{\mathcal{Q}}+\frac{1}{\sqrt{\xi}}(\widetilde{\mathcal{Q}} \eta(L)+\eta(L) \widetilde{\mathcal{Q}}) \quad(\text { Stratonovich })
$$

This equation is a central result that will be used below.

\section{Matrix generalization of the Dufresne identity}

In this section, we discuss the relation with the work of Rider and Valkó [60] and extend their result. We have obtained the symmetrised Wigner-Smith matrix under the form of an exponential functional of the matrix BM (60). A first difference with Rider and Valkó's functional concerns the form of the integral. A second difference is that the matrix BM of Rider and Valkó involves a non Hermitian noise with $N^{2}$ independent real entries (orthogonal class), while we have considered a Hermitian real or complex noise (orthogonal or unitary class).

\subsection{Exponential functional}

Let us introduce

$$
\Lambda(x / \xi)^{\dagger}=X(L) X(L-x)^{-1}, \quad x \in[0, L] .
$$

From (91), we get that $\Lambda$ satisfies the MSDE

$$
\partial_{x} \Lambda=-\mu \Lambda+\eta(x) \Lambda
$$

where $\eta(x)$ is the Hermitian Gaussian white noise and $\mu=1+\beta(N-1) / 2$. The initial condition is obviously $\Lambda(0)=\mathbf{1}_{N}$. The representation (60) can be rewritten in a more simple form with the new matricial random process:

$$
\widetilde{\mathcal{Q}} \stackrel{\text { (law) }}{=} 2 \tau_{\xi} \int_{0}^{L / \xi} \mathrm{d} x \Lambda(x)^{\dagger} \Lambda(x)
$$




\section{2. 'Gauge' transformation}

Rider and Valkó in reference [60] have considered the matricial stochastic process

$$
\partial_{x} M(x)=-\mu M(x)+\chi(x) M(x),
$$

where $\chi(x)$ is an $N \times N$ real matrix, whose elements are $N^{2}$ independent normalised real Gaussian white noises. Thus the noise matrix is non Hermitian, $\chi(x) \neq \chi(x)^{\dagger}$. Let us rather consider (96) when the matrix elements of $\chi(x)$ are $N^{2}$ independent complex noises. We decompose it into Hermitian and anti-Hermitian parts:

$$
\chi=\eta+\mathrm{i} A, \quad \eta=\frac{\chi+\chi^{\dagger}}{2}, \quad A=\frac{\chi-\chi^{\dagger}}{2 \mathrm{i}},
$$

thus $\left\langle\eta_{a b}(x) \eta_{c d}\left(x^{\prime}\right)\right\rangle=C_{a b, c d} \delta\left(x-x^{\prime}\right)$. In order to relate (96) to (94), we 'gauge out' the nonHermitian part

$$
M=U \Lambda \quad \text { where } \quad \partial_{x} U=\mathrm{i} A U .
$$

It is straightforward to get

$$
\partial_{x} \Lambda(x)=-\mu \Lambda(x)+U(x)^{-1} \eta(x) U(x) \Lambda(x) .
$$

These equations are understood in the Stratonovich convention. We use the mathematical notation $\mathrm{d} \Lambda=-\mu \Lambda \mathrm{d} x+U^{-1} \mathrm{~d} B(x) U \Lambda$, where $\mathrm{d} B(x)=\eta(x) \mathrm{d} x$. Let us now go to the Itô convention. Using equation (64), we get

$$
\mathrm{d} \Lambda=-\frac{\mu}{2} \Lambda \mathrm{d} x+U^{-1} \mathrm{~d} B(x) U \Lambda \stackrel{\text { (law) }}{=}-\frac{\mu}{2} \Lambda \mathrm{d} x+\mathrm{d} B(x) \Lambda \quad \text { (Itô). }
$$

Now going back to the Stratonovich convention, we deduce $\mathrm{d} \Lambda=-\mu \Lambda \mathrm{d} x+\mathrm{d} B(x) \Lambda$, i.e. equation (94).

\subsection{Matrix Dufresne identity}

Using the connection with the MSDE studied in reference [61], we generalize in this paragraph Rider and Valkó's result for $\beta=1$ to both symmetry classes $(\beta=1$ and 2 ). The matricial process studied in reference [61], which arises in a different multichannel localization model, is

$$
\partial_{x} \mathcal{Z}=|\varepsilon|^{2}-2 \mu g \mathcal{Z}-\mathcal{Z}^{2}+\sqrt{g}[\eta(x) \mathcal{Z}+\mathcal{Z} \eta(x)]
$$

It was shown to be characterized by the stationary matrix distribution (in the $x \rightarrow \infty$ limit)

$$
P(\mathcal{Z}) \propto(\operatorname{det} \mathcal{Z})^{-1-\mu-\beta(N-1) / 2} \exp \left[-\frac{1}{2 g} \operatorname{tr}\left\{\mathcal{Z}+|\varepsilon|^{2} \mathcal{Z}^{-1}\right\}\right]
$$

The distribution was obtained from the analysis of the related matrix Fokker-Planck equation (the case where the noise $\eta$ is not isotropic was also considered in reference [61]). The mapping to our problem can be easily realised: we set

$$
\mathcal{Z} \rightarrow k|\varepsilon|^{2} \widetilde{\mathcal{Q}} \quad \text { and } \quad g \rightarrow 1 / \xi
$$

in equations (101) and (102). For $\mu>\beta(N-1) / 2$, it is possible to take the limit $|\varepsilon| \rightarrow 0$ and recover the MSDE (92) while the distribution (102) takes the form

$$
P(\widetilde{\mathcal{Q}}) \propto(\operatorname{det} \widetilde{\mathcal{Q}})^{-1-\mu-\beta(N-1) / 2} \mathrm{e}^{-\tau_{\xi} \operatorname{tr}\left\{\widetilde{\mathcal{Q}}^{-1}\right\}} .
$$


The change of variable $\Gamma=2 \tau_{\xi} \widetilde{\mathcal{Q}}^{-1}$ leads to the Wishart distribution

$$
P(\Gamma)=C_{N, \beta}(\operatorname{det} \Gamma)^{\mu-1-\beta(N-1) / 2} \mathrm{e}^{-(1 / 2) \operatorname{tr}\{\Gamma\}} \text { for } \mu>\frac{\beta(N-1)}{2}
$$

where $C_{N, \beta}$ is a normalisation. Having shown the relation between the MSDE (92) and the representation $(95)$, we conclude that

$$
\left(\int_{0}^{\infty} \mathrm{d} x M(x)^{\dagger} M(x)\right)^{-1}=\left(\int_{0}^{\infty} \mathrm{d} x \Lambda(x)^{\dagger} \Lambda(x)\right)^{-1} \stackrel{(\text { law })}{=} \Gamma,
$$

is distributed according to (105), where $\Lambda(x)$ solves (95) for a Hermitian noise and $M(x)$ solves (96) for a non Hermitian noise. Here, the derivation was done for arbitrary drift $\mu$, thus extending the Dufresne identity to the case of matrix BM, for orthogonal and unitary classes. Coming back to the multichannel disordered wire model, the drift is given by (19), leading to (10), i.e. to Beenakker \& Brouwer's result (9).

\section{Application: moments $\langle\operatorname{tr}\{\mathcal{Q}\}\rangle,\left\langle\operatorname{tr}\{\mathcal{Q}\}^{2}\right\rangle$ and $\left\langle\operatorname{tr}\left\{\mathcal{Q}^{2}\right\}\right\rangle$}

In this section we show that the representation (95) in terms of exponential functional of the matrix BM, or equivalently the MSDE (92), can be used to compute the moments of the form $\left\langle\operatorname{tr}\left\{\mathcal{Q}^{n}\right\}^{m}\right\rangle$. We consider the first moments. Our starting point is to rewrite the MSDE (92) in the Itô convention. We use the notation $\mathrm{d} B(x)=\eta(x) \mathrm{d} x$ as in the previous section. Using (63) and (64), we get:

$$
\mathrm{d} \widetilde{\mathcal{Q}}=\left(\frac{\mathbf{1}_{N}}{k}+\frac{\beta}{2 \xi}\left[\operatorname{tr}\{\widetilde{\mathcal{Q}}\} \mathbf{1}_{N}-N \widetilde{\mathcal{Q}}\right]\right) \mathrm{d} x+\frac{1}{\sqrt{\xi}}(\widetilde{\mathcal{Q}} \mathrm{d} B(x)+\mathrm{d} B(x) \widetilde{\mathcal{Q}}) \text { (Itô) }
$$

We deduce immediately the equation for the trace

$$
\operatorname{dtr}\{\widetilde{\mathcal{Q}}\}=\frac{N}{k} \mathrm{~d} x+\frac{2}{\sqrt{\xi}} \operatorname{tr}\{\widetilde{\mathcal{Q}} \mathrm{d} B(x)\} \quad \text { (Itô) }
$$

and thus

$$
\mathrm{d}\langle\operatorname{tr}\{\mathcal{Q}\}\rangle=\frac{N}{k} \mathrm{~d} x
$$

where we have used $\operatorname{tr}\left\{\widetilde{\mathcal{Q}}^{n}\right\}=\operatorname{tr}\left\{\mathcal{Q}^{n}\right\}$. After integration over $[0, L]$, we recover the expected behaviour, equation (12),

$$
\langle\operatorname{tr}\{\mathcal{Q}\}\rangle=\frac{N L}{k}=\frac{2 N L}{v},
$$

where $v=2 k$ is the group velocity.

Next, we write an equation for $[\operatorname{tr}\{\widetilde{\mathcal{Q}}\}]^{2}$. Using Itô's formula ${ }^{9}$ we have

$$
\mathrm{d}\left(\operatorname{tr}\{\widetilde{\mathcal{Q}}\}^{2}\right)=2 \operatorname{tr}\{\widetilde{\mathcal{Q}}\} \operatorname{dtr}\{\widetilde{\mathcal{Q}}\}+(\operatorname{dtr}\{\widetilde{\mathcal{Q}}\})^{2}
$$

\footnotetext{
${ }^{9}$ Using the notations of the footnote 6, a simple way to recover Itô's formula is as follows: $\mathrm{d} f(x)=f(x+\mathrm{d} x)-$ $f(x)=f^{\prime}(x) \mathrm{d} x+\frac{1}{2} f^{\prime \prime}(x) \mathrm{d} x^{2}=f^{\prime}(x) \mathrm{d} x+\frac{1}{2} f^{\prime \prime}(x)\left[(\mathrm{d} x)_{\text {noise }}\right]^{2}$. This form is appropriate to be applied to the case of MSDE.
} 
Using

$$
(\operatorname{tr}\{\widetilde{\mathcal{Q}} \mathrm{d} B(x)\})^{2}=\operatorname{tr}\left\{\widetilde{\mathcal{Q}}^{2}\right\} \mathrm{d} x
$$

we deduce

$$
\operatorname{dtr}\{\widetilde{\mathcal{Q}}\}^{2}=\left[\frac{2 N}{k} \operatorname{tr}\{\widetilde{\mathcal{Q}}\}+\frac{4}{\xi} \operatorname{tr}\left\{\widetilde{\mathcal{Q}}^{2}\right\}\right] \mathrm{d} x+\frac{4}{\sqrt{\xi}} \operatorname{tr}\{\widetilde{\mathcal{Q}}\} \operatorname{tr}\{\widetilde{\mathcal{Q}} \mathrm{d} B(x)\} \text { (Itô) }
$$

This makes clear that we have to derive also an equation for $\operatorname{tr}\left\{\widetilde{\mathcal{Q}}^{2}\right\}$. From (107), the application of the Itô formula gives

$$
\begin{aligned}
\mathrm{d}\left(\widetilde{\mathcal{Q}}^{2}\right)= & \left\{\frac{2 \widetilde{\mathcal{Q}}}{k}+\frac{1}{\xi}\left[\frac{\beta}{2} \operatorname{tr}\left\{\widetilde{\mathcal{Q}}^{2}\right\} \mathbf{1}_{N}+2 \beta \operatorname{tr}\{\widetilde{\mathcal{Q}}\} \widetilde{\mathcal{Q}}+\left(2(2-\beta)-\frac{\beta N}{2}\right) \widetilde{\mathcal{Q}}^{2}\right]\right\} \mathrm{d} x \\
& +\frac{1}{\sqrt{\xi}}\left[\widetilde{\mathcal{Q}}^{2} \mathrm{~d} B(x)+2 \widetilde{\mathcal{Q}} \mathrm{d} B(x) \widetilde{\mathcal{Q}}+\mathrm{d} B(x) \widetilde{\mathcal{Q}}^{2}\right] \quad \text { (Itô) }
\end{aligned}
$$

A trace gives

$$
\begin{aligned}
\operatorname{dtr}\left\{\widetilde{\mathcal{Q}}^{2}\right\}= & \left(\frac{2}{k} \operatorname{tr}\{\widetilde{\mathcal{Q}}\}+\frac{4}{\xi}\left[\frac{\beta}{2} \operatorname{tr}\{\widetilde{\mathcal{Q}}\}^{2}+\left(1-\frac{\beta}{2}\right) \operatorname{tr}\left\{\widetilde{\mathcal{Q}}^{2}\right\}\right]\right) \mathrm{d} x \\
& +\frac{4}{\sqrt{\xi}} \operatorname{tr}\left\{\widetilde{\mathcal{Q}}^{2} \mathrm{~d} B(x)\right\} \quad \text { (Itô). }
\end{aligned}
$$

Averaging (113) and (115), we deduce a simple linear problem

$$
\frac{\partial}{\partial L}\left(\begin{array}{c}
\left\langle\operatorname{tr}\{\mathcal{Q}\}^{2}\right\rangle \\
\left\langle\operatorname{tr}\left\{\mathcal{Q}^{2}\right\}\right\rangle
\end{array}\right)=\frac{2 N L}{k^{2}}\left(\begin{array}{c}
N \\
1
\end{array}\right)+\frac{4}{\xi}\left(\begin{array}{cc}
0 & 1 \\
\frac{\beta}{2} & 1-\frac{\beta}{2}
\end{array}\right)\left(\begin{array}{c}
\left\langle\operatorname{tr}\{\mathcal{Q}\}^{2}\right\rangle \\
\left\langle\operatorname{tr}\left\{\mathcal{Q}^{2}\right\}\right\rangle
\end{array}\right)
$$

It is useful to use the spectral decomposition

$$
M_{\beta}=\left(\begin{array}{cc}
0 & 1 \\
\frac{\beta}{2} & 1-\frac{\beta}{2}
\end{array}\right)=\sum_{\sigma= \pm} \lambda_{\sigma} \Pi_{\sigma}
$$

where the eigenvalues $\lambda_{ \pm}$and the corresponding projectors are

$$
\begin{aligned}
& \lambda_{+}=1 \quad \text { and } \quad \Pi_{+}=\frac{1}{1+\frac{\beta}{2}}\left(\begin{array}{cc}
\frac{\beta}{2} & 1 \\
\frac{\beta}{2} & 1
\end{array}\right) \\
& \lambda_{-}=-\frac{\beta}{2} \quad \text { and } \quad \Pi_{-}=\frac{1}{1+\frac{\beta}{2}}\left(\begin{array}{cc}
1 & -1 \\
-\frac{\beta}{2} & \frac{\beta}{2}
\end{array}\right) .
\end{aligned}
$$

Integration of (116) gives

$$
\left(\begin{array}{c}
\left\langle\operatorname{tr}\{\mathcal{Q}\}^{2}\right\rangle \\
\left\langle\operatorname{tr}\left\{\mathcal{Q}^{2}\right\}\right\rangle
\end{array}\right)=\frac{2 N}{k^{2}} \int_{0}^{L} \mathrm{~d} x \times \mathrm{e}^{\frac{4}{\xi} M_{\beta}(L-x)}\left(\begin{array}{c}
N \\
1
\end{array}\right) .
$$


Using $\mathrm{e}^{M_{\beta} y}=\sum_{\sigma} \mathrm{e}^{\lambda_{\sigma} y} \Pi_{\sigma}$, some simple algebra gives

$$
\begin{aligned}
& \left\langle\operatorname{tr}\{\mathcal{Q}\}^{2}\right\rangle=\frac{N \tau_{\xi}^{2}}{2}\left\{\frac{1+\frac{\beta N}{2}}{1+\frac{\beta}{2}}\left[\mathrm{e}^{4 L / \xi}-1-\frac{4 L}{\xi}\right]+\frac{\left(\frac{2}{\beta}\right)^{2}(N-1)}{1+\frac{\beta}{2}}\left[\mathrm{e}^{-2 \beta L / \xi}-1+\frac{2 \beta L}{\xi}\right]\right\} \\
& \left\langle\operatorname{tr}\left\{\mathcal{Q}^{2}\right\}\right\rangle=\frac{N \tau_{\xi}^{2}}{2}\left\{\frac{1+\frac{\beta N}{2}}{1+\frac{\beta}{2}}\left[\mathrm{e}^{4 L / \xi}-1-\frac{4 L}{\xi}\right]-\frac{\frac{2}{\beta}(N-1)}{1+\frac{\beta}{2}}\left[\mathrm{e}^{-2 \beta L / \xi}-1+\frac{2 \beta L}{\xi}\right]\right\}
\end{aligned}
$$

For $N=1$, we recover $\left\langle\mathcal{Q}^{2}\right\rangle=\frac{\tau_{\xi}^{2}}{2}\left(\mathrm{e}^{4 L / \xi}-1-\frac{4 L}{\xi}\right)$ [29], as it should.

We remark that

$$
\left\langle\operatorname{tr}\{\mathcal{Q}\}^{2}\right\rangle-\left\langle\operatorname{tr}\left\{\mathcal{Q}^{2}\right\}\right\rangle=\tau_{\xi}^{2} N(N-1) \frac{4}{\beta}\left[\frac{L}{\xi}+\frac{\mathrm{e}^{-2 \beta L / \xi}-1}{2 \beta}\right] .
$$

Introducing the proper time delays $\left\{\tau_{a}\right\}_{a=1, \ldots, N}$, the eigenvalues of the Wigner-Smith matrix (see [10]), we see that (123) corresponds to $\sum_{a \neq b}\left\langle\tau_{a} \tau_{b}\right\rangle$ and hence provides some information about the correlations between proper times.

These expressions can be used to obtain the statistical properties of the proper time delays. In the limit of long disordered region, $L \gg \xi$, the fact that $\left\langle\operatorname{tr}\{\mathcal{Q}\}^{2}\right\rangle \simeq\left\langle\operatorname{tr}\left\{\mathcal{Q}^{2}\right\}\right\rangle$ shows that proper times are weaky correlated. Using that all channels are equivalent, we get the mean value

$$
\left\langle\tau_{a}\right\rangle=\frac{L}{k}=2 \tau_{\xi} \frac{L}{\xi}
$$

the second moment $\left\langle\tau_{a}^{2}\right\rangle=(1 / N)\left\langle\operatorname{tr}\left\{\mathcal{Q}^{2}\right\}\right\rangle$

$$
\left\langle\tau_{a}^{2}\right\rangle \simeq \tau_{\xi}^{2} \frac{N \beta}{2(\beta+2)} \mathrm{e}^{4 L / \xi}
$$

and from (123), the correlation

$$
\left\langle\tau_{a} \tau_{b}\right\rangle=\tau_{\xi}^{2} \frac{4}{\beta}\left[\frac{L}{\xi}+\frac{\mathrm{e}^{-2 \beta L / \xi}-1}{2 \beta}\right] \simeq \tau_{\xi}^{2} \frac{4 L}{\beta \xi} \text { for } a \neq b,
$$

demonstrating that, in disordered multichannel wires, the proper times are characterized by weak anti-correlation $\operatorname{Cov}\left(\tau_{a}, \tau_{b}\right) \simeq-\left(2 \tau_{\xi} L / \xi\right)^{2}$.

\section{Resolvent}

\subsection{Resolvent and density of eigenvalues}

We re-examine the problem considered by Ossipov [45], within our disordered model. From the MSDE (92), we can deduce an equation for the evolution of the density of eigenvalues of the matrix $\widetilde{\mathcal{Q}}$, and thus of $\mathcal{Q}$. We introduce the resolvent matrix

$$
G(z ; L)=\left(z \mathbf{1}_{N}-\frac{N}{2 \tau_{\xi}} \widetilde{\mathcal{Q}}(L)\right)^{-1} .
$$


The matrix $\widetilde{\mathcal{Q}}$ is rescaled by a factor $N$, as we expect from the distribution (10) that its eigenvalues scale as $\mathcal{O}\left(N^{-1}\right)$ for large number of channels. From the MSDE (92), we can write the equation satisfied by $G(z ; L)$ :

$$
\partial_{L} G=\frac{N}{\xi} G^{2}-\frac{2 \mu}{\xi} G\left(z G-\mathbf{1}_{N}\right)+\frac{1}{\sqrt{\xi}}\left[\left(z G-\mathbf{1}_{N}\right) \eta(L) G+G \eta(L)\left(z G-\mathbf{1}_{N}\right)\right],
$$

where we have omitted the arguments of $G$ for clarity. Writing $\mathrm{d} B(x)=\eta(x) \mathrm{d} x$ along with the relations (64) and (63), we can convert this equation to the Itô convention. We get

$$
\begin{aligned}
\partial_{L} G= & \frac{N}{\xi} G^{2}-\frac{2 \mu}{\xi} G,\left(z G-\mathbf{1}_{N}\right) \\
& +\frac{1}{\xi}\left(2 z G-\mathbf{1}_{N}\right)\left[-\mu G+\left(2 z G-\mathbf{1}_{N}\right)\left(\frac{\beta}{2} \operatorname{tr}\{G\}+\left(1-\frac{\beta}{2}\right) G\right)\right] \\
& +\frac{1}{\sqrt{\xi}}\left[\left(z G-\mathbf{1}_{N}\right) \eta(L) G+G \eta(L)\left(z G-\mathbf{1}_{N}\right)\right] \quad \text { (Itô). }
\end{aligned}
$$

We can now take the expectation value, which yields

$$
\begin{aligned}
\partial_{L}\langle G\rangle= & \frac{N}{\xi}\left\langle G^{2}\right\rangle-\frac{4 \mu z}{\xi}\left\langle G^{2}\right\rangle+\frac{3 \mu}{\xi}\langle G\rangle+\frac{\beta}{2 \xi}\left(\langle\operatorname{tr}\{G\}\rangle-4 z\langle G \operatorname{tr}\{G\}\rangle+4 z^{2}\left\langle G^{2} \operatorname{tr}\{G\}\right\rangle\right) \\
& +\frac{1}{\xi}\left(1-\frac{\beta}{2}\right)\left(\langle G\rangle-4 z\left\langle G^{2}\right\rangle+4 z^{2}\left\langle G^{3}\right\rangle\right) .
\end{aligned}
$$

Using that $\partial_{z} G(z)=-G(z)^{2}$ and $\partial_{z}^{2} G(z)=2 G(z)^{3}$, we can write

$$
\begin{aligned}
\partial_{L}\langle G\rangle= & -\frac{N}{\xi} \partial_{z}\langle G\rangle+\frac{4 \mu z}{\xi} \partial_{z}\langle G\rangle+\frac{3 \mu}{\xi}\langle G\rangle+\frac{\beta}{2 \xi}(\langle\operatorname{tr}\{G\}\rangle-4 z\langle G \operatorname{tr}\{G\}\rangle \\
& \left.-4 z^{2}\left\langle\left(\partial_{z} G\right) \operatorname{tr}\{G\}\right\rangle\right)+\frac{1}{\xi}\left(1-\frac{\beta}{2}\right)\left(\langle G\rangle+4 z\left\langle\partial_{z} G\right\rangle+2 z^{2}\left\langle\partial_{z}^{2} G\right\rangle\right) .
\end{aligned}
$$

In the limit $N \rightarrow \infty$, it is expected that the resolvent

$$
g(z ; L)=\frac{1}{N} \operatorname{tr}\{G(z ; L)\}
$$

becomes deterministic, so we can take it out of the expectation values. Hence

$$
\begin{aligned}
\partial_{L}\langle G\rangle= & -\frac{N}{\xi} \partial_{z}\langle G\rangle+\frac{4 \mu z}{\xi} \partial_{z}\langle G\rangle+\frac{3 \mu}{\xi}\langle G\rangle+\frac{\beta}{2 \xi}\left(N g-4 N z g\langle G\rangle-4 N z^{2} g\left\langle\partial_{z} G\right\rangle\right) \\
& +\frac{1}{\xi}\left(1-\frac{\beta}{2}\right)\left(\langle G\rangle+4 z\left\langle\partial_{z} G\right\rangle+2 z^{2}\left\langle\partial_{z}^{2} G\right\rangle\right) .
\end{aligned}
$$

Taking the trace of this equation, and keeping only the leading order terms in $N$ for consistency, we deduce a partial differential equation for $g(z ; L)$ :

$$
\frac{\partial g(z ; L)}{\partial L}=\frac{N}{\xi} \frac{\partial}{\partial z}\left(2\left(\beta z-\frac{1}{2}\right) g(z ; L)-\beta z^{2} g(z ; L)^{2}\right)
$$

The density of eigenvalues $\rho(\lambda ; L)$ can then be obtained from $g(z ; L)$ as

$$
\rho(\lambda ; L)=-\frac{1}{\pi} \operatorname{Im}\left[g\left(\lambda+\mathrm{i} 0^{+} ; L\right)\right] .
$$


In order to connect our equations with the result of reference [45], we should have kept the last subleading term in (133), leading then to

$$
\frac{\partial g(z ; L)}{\partial L}=\frac{1}{\xi} \frac{\partial}{\partial z}\left[N\left(2\left(\beta z-\frac{1}{2}\right) g(z ; L)-\beta z^{2} g(z ; L)^{2}\right)+(2-\beta) \frac{\partial}{\partial z}\left(z^{2} g(z ; L)\right)\right] .
$$

For $\beta=1$, equation (136) exactly coincides with equation (12) of reference [45] (upon rescaling $z \rightarrow z / 4$ and hence $g \rightarrow 4 g$, due to a different definition). A nice solution of equation (136) was obtained by using the analogy with the Burgers equation.

\subsection{Discussion}

In his article [45], Ossipov has claimed that his 'approach provides (...) foundation for the arguments of the scaling theory of Anderson localization', and that 'scattering isotropy (...) is not used in our approach', which 'allows to study the problem in higher dimension'. We disagree with these statements.

For $\beta=1$, one can write $\mathcal{Q}=\mathcal{O} \operatorname{diag}\left(\tau_{1}, \ldots, \tau_{N}\right) \mathcal{O}^{\mathrm{T}}$, where the orthogonal matrix $\mathcal{O}$ gathers the eigenvectors of the Wigner-Smith matrix. Ossipov has argued that the derivation of his equation (12), i.e. (136) for $\beta=1$, relies on assumptions that $\mathcal{S}$ and $\mathcal{O}$ would be controlled by fast variables, while the eigenvalues $\left\{\tau_{1}, \ldots, \tau_{N}\right\}$ would be the only slow variables. First, let us stress that reference [45] (and its supplementary material) only provides statistical information on the moments or correlations of the elements of two matrices $\mathcal{S}$ and $\mathcal{O}$. The correlations between eigenvalues and eigenvectors of $\mathcal{Q}$ were not investigated in reference [45], nor the relaxation of the variables. Second, matrices are characterised by many degree of freedom, thus a clear separation of fast and slow variables may be difficult, even numerically. Third, in our paper, working with $\widetilde{\mathcal{S}}$ instead of the scattering matrix $\mathcal{S}$ has eliminated the fast variables. This has led to matrix stochastic differential equations for $\widetilde{\mathcal{S}}$ and the symmetrised Wigner-Smith matrix $\widetilde{\mathcal{Q}}$. Using the isotropy assumption, these MSDE have been decoupled, leading to the matrix $\operatorname{SDE}(92)$ for $\widetilde{\mathcal{Q}}$. Thus we have obtained that the matrix $\widetilde{\mathcal{Q}}$ is controlled by slow variables, i.e. both its eigenvalues and eigenvectors are slow variables. Their decoupling relies on the isotropy assumption.

The fact that we have recovered Ossipov's equation within a model based on isotropy assumption, emphasizes that Ossipov's central equation (12) has the same physical content as the equation obtained within the DMPK approach (Ossipov agrees that his 'equation (8) [from which his equation (12) is derived] coincides with the DMPK equation'). These equations describe disordered wires which are transversally ergodic, i.e. are inherently restricted to the quasi-one-dimensional regime and cannot encode the physics of Anderson localisation in dimension $d \geqslant 2$.

\section{Conclusion}

We have studied the Wigner-Smith time delay matrix $\mathcal{Q}$ for multichannel disordered wires of length $L$. Using an isotropy assumption, we have been able to use the decoupling between fast and slow variables both for the scattering matrix and the Wigner-Smith matrix (the two length scales associated with fast and slow variables are the wavelength $\lambda$ and the elastic mean free path $\ell_{\mathrm{e}}$, respectively). For this purpose, the new symmetrisation of the Wigner-Smith matrix was crucial. We have provided some matrix stochastic differential equations for matrices controlling the slow variables, which have eventually led to a representation of $\widetilde{\mathcal{Q}}$, the symmetrised $\mathcal{Q}$-matrix, under the form of an exponential functional of a matrix Brownian motion (BM). This 
representation is a generalization of the result for $N=1$, obtained by one of us with Comtet $[29,31]$.

We stress that our approach is based on the analysis of a matricial random process (like in reference [61]), rather that a derivation of a Fokker-Planck equation for the eigenvalues, like in the standard DMPK approach.

In the limit of semi-infinite disordered region, $L \rightarrow \infty$, by making use of an extension of the recent matrix Dufresne identity of Rider and Valkó [60], we have recovered straightforwardly the distribution of the $\mathcal{Q}$ 's eigenvalues found by Brouwer and Beenakker $[46,47]$ by different means.

Furthermore, our exponential functional representation allows to study the statistical properties of $\mathcal{Q}$ for finite $L$ : we have derived the first moments. In particular, we have shown that $\left\langle\operatorname{tr}\left\{\mathcal{Q}^{2}\right\}\right\rangle$ and $\left\langle\operatorname{tr}\{\mathcal{Q}\}^{2}\right\rangle$ both behave as $\sim \exp [4 L / \xi]$. The structure of the calculation (with the result for $N=1$ of reference [29]) suggests to conjecture the form

$$
\left\langle\operatorname{tr}\left\{\mathcal{Q}^{n_{1}}\right\} \operatorname{tr}\left\{\mathcal{Q}^{n_{2}}\right\} \cdots \operatorname{tr}\left\{\mathcal{Q}^{n_{k}}\right\}\right\rangle \sim \mathrm{e}^{2 n(n-1) L / \xi} \quad \text { where } n=\sum_{i=1}^{k} n_{i} .
$$

in the general case

In section 2, we have followed an alternative approach for the derivation of the exponential functional representation of the BM. Although this derivation was less rigorous, it emphasizes the universal character of the results. Interestingly, it suggests that $\mathcal{Q}$ has also a representation in terms of exponential functional of the BM. This point still deserves some clarifications.

Finally, our analysis opens the natural question of finding extensions of our results. Elimination of fast variables relies on a high energy/disorder and some isotropy assumption (invariance between exchange of channels). Would it be possible to relax the second hypothesis ? Is there an exponential functional of the BM representation for non identical channels ? For example by considering the case of channel dependent wave vectors, $k \mathbf{1}_{N} \rightarrow \operatorname{diag}\left(k_{1}, \ldots, k_{N}\right)$, and/or anisotropic correlations $C_{a b, c d}$, which are relevant to describe more realistic mutlichannel disordered wires.

\section{Acknowledgments}

We thank Alain Comtet for stimulating discussions. This project has benefitted from funding of the Netherlands Organization for Scientific Research (NWO/OCW) and from the European Research Council (ERC) under the European Union's Horizon 2020 research and innovation programme.

\section{Appendix A. Wigner-Smith matrix in terms of the stationary scattering states: derivation of equation (27)}

A relation similar to equation (27) was derived by Friedel [62] and Smith [8] for centrosymmetric potential. A proof for the more general case of metric graphs was given in references $[63,64]$ (cf equations (43) and (53) of the first reference, or equation (14) of the second). Here, we briefly adapt the derivation of reference [65] for metric graphs to the case of multichannel disordered wires. We consider $\Phi=\sqrt{h v} \Psi$, which presents the 'asymptotic' behaviour

$$
\Phi(x)=\mathbf{1}_{N} \mathrm{e}^{-\mathrm{i} k(x-L)}+\mathcal{S}(\varepsilon) \mathrm{e}^{\mathrm{i} k(x-L)} \quad \text { for } x \geqslant L .
$$


Step 1: let us define the $N \times N$ matrix

$$
\Omega(x) \stackrel{\text { def }}{=} \frac{\partial \Phi^{\dagger}}{\partial x} \frac{\partial \Phi}{\partial \varepsilon}-\Phi^{\dagger} \frac{\partial^{2} \Phi}{\partial x \partial \varepsilon}
$$

which satisfies

$$
\frac{\partial \Omega(x)}{\partial x}=\Phi^{\dagger}(x) \Phi(x)
$$

Step 2: we compute $\Omega(x)$ at the boundaries. Dirichlet boundary condition gives $\Omega(0)=0$. Using the expression of the scattering state (A.1), we find

$$
\Omega(L)=-2 \mathrm{i} k \mathcal{S}^{\dagger} \frac{\partial \mathcal{S}}{\partial \varepsilon}-\frac{\mathrm{i}}{2 k}\left(\mathcal{S}-\mathcal{S}^{\dagger}\right)
$$

Step 3: we combine

$$
\int_{0}^{L} \mathrm{~d} x \frac{\partial \Omega(x)}{\partial x}=\Omega(L)-\Omega(0)
$$

with (A.3) and (A.4). Multiplication by $1 /(4 \pi k)$ we end with the matricial identity (27).

Equation (27) and the DoS-Note that the trace $\rho(x ; \varepsilon)=\operatorname{tr}\left\{\Psi_{\varepsilon}^{\dagger}(x) \Psi_{\varepsilon}(x)\right\}$ has the interpretation of the local density of states, thus we recover the Krein-Friedel relation [63-65]

$$
\int_{0}^{L} \mathrm{~d} x \rho(x ; \varepsilon)=\frac{1}{2 \pi} \operatorname{tr}\{\mathcal{Q}\}+\frac{\operatorname{tr}\left\{\mathcal{S}-\mathcal{S}^{\dagger}\right\}}{8 \mathrm{i} \pi \varepsilon} .
$$

\section{Appendix B. Effective MSDE from the Fokker-Planck equation for $\mathbf{N}=\mathbf{2}$ and} $\beta=1$

In this appendix, we show a rigorous procedure to average over the fast variables based on the Fokker-Planck equation. Let us consider for simplicity $N=2$ and $\beta=1$. We take a noise of the form

$$
V=\left(\begin{array}{cc}
\sqrt{\sigma_{1}} v_{1} & \sqrt{\sigma_{2} / 2} v_{2} \\
\sqrt{\sigma_{2} / 2} v_{2} & \sqrt{\sigma_{3}} v_{3}
\end{array}\right),
$$

where $v_{1}, v_{2}$ and $v_{3}$ are independent Gaussian white noises of unit variance. Setting $\sigma_{1}=\sigma_{2}=$ $\sigma_{3}=\sigma$ corresponds to the isotropic case (15).

We parametrise the scattering matrix in terms of its eigenvalues and eigenvectors

$$
\mathcal{S}=\mathcal{O}^{\mathrm{T}} \mathrm{e}^{2 \mathrm{i} \Phi} \mathcal{O}
$$

where

$$
\Phi=\operatorname{Diag}\left(\phi_{1}, \phi_{2}\right)
$$

and $\mathcal{O}$ is a rotation matrix:

$$
\mathcal{O}=\left(\begin{array}{cc}
\cos \theta & -\sin \theta \\
\sin \theta & \cos \theta
\end{array}\right) .
$$


Using this parametrisation in equation (40), we obtain the equations for $\phi_{1}, \phi_{2}$ and $\theta$ :

$$
\frac{\mathrm{d}}{\mathrm{d} x}\left(\begin{array}{c}
\phi_{1} \\
\phi_{2} \\
\theta
\end{array}\right)=\left(\begin{array}{l}
k \\
k \\
0
\end{array}\right)+\frac{1}{k} b\left(\phi_{1}, \phi_{2}, \theta\right)\left(\begin{array}{l}
v_{1} \\
v_{2} \\
v_{3}
\end{array}\right) \quad \text { (Stratonovich) }
$$

where $b$ is the following $3 \times 3$ matrix

$$
\left(\begin{array}{ccc}
-\sqrt{\sigma_{1}} \cos ^{2} \theta \cos ^{2} \phi_{1} & \frac{\sqrt{\sigma_{2}}}{\sqrt{2}} \sin (2 \theta) \cos ^{2} \phi_{1} & -\sqrt{\sigma_{3}} \sin ^{2} \theta \cos ^{2} \phi_{1} \\
-\sqrt{\sigma_{1}} \sin ^{2} \theta \cos ^{2} \phi_{2} & -\frac{\sqrt{\sigma_{2}}}{\sqrt{2}} \sin (2 \theta) \cos ^{2} \phi_{2} & -\sqrt{\sigma_{3}} \cos ^{2} \theta \cos ^{2} \phi_{2} \\
\frac{\sqrt{\sigma_{1}} \cos \phi_{1} \cos \phi_{2} \sin (2 \theta)}{2 \sin \left(\phi_{1}-\phi_{2}\right)} & \frac{\sqrt{\sigma_{2}} \cos (2 \theta) \cos \phi_{1} \cos \phi_{2}}{\sqrt{2} \sin \left(\phi_{1}-\phi_{2}\right)} & -\sqrt{\sigma_{3}} \frac{\cos \phi_{1} \cos \phi_{2} \sin (2 \theta)}{2 \sin \left(\phi_{1}-\phi_{2}\right)}
\end{array}\right)
$$

Since the two phases evolve rapidly (on the scale $1 / k$ ), we denote

$$
\phi_{1}=k x+\tilde{\phi}_{1}, \quad \phi_{2}=k x+\tilde{\phi}_{2} .
$$

Our aim is to obtain equations describing the evolution of $\tilde{\phi}_{1}, \tilde{\phi}_{2}$, and $\theta$. We will average over the fast variables $k x$, at the level of the Fokker-Planck equation

$$
\frac{\partial P}{\partial x}\left(\tilde{\phi}_{1}, \tilde{\phi}_{2}, \theta\right)=\frac{1}{2} \sum_{i, j, l=1}^{3} \frac{\partial}{\partial X_{i}} b_{i l} \frac{\partial}{\partial X_{j}} b_{j l} P
$$

where $X=\left(\tilde{\phi}_{1}, \tilde{\phi}_{2}, \theta\right)$. The idea is to average over $k x$ on one period (the slow variables $\tilde{\phi}_{1}, \tilde{\phi}_{2}$, and $\theta$ can be considered constant on this scale). For this, we need all the derivatives to be in the front (which corresponds to converting the SDEs to Itô). Then, after averaging, we obtain an equation of the form

$$
\frac{\partial P}{\partial x}\left(\tilde{\phi}_{1}, \tilde{\phi}_{2}, \theta\right)=\sum_{i, j}^{3} \frac{\partial}{\partial X_{i}}\left(-\tilde{a}_{i}+\frac{1}{2} \frac{\partial}{\partial X_{j}} \tilde{c}_{i j}\right) P
$$

which we can interpret as a new Fokker-Planck equation, with a drift $\tilde{a}$ (in Itô) and a new matrix $\tilde{c}$ which we need to decompose into the form $\tilde{c}=\tilde{b} \tilde{b}^{\mathrm{T}}$ in order to write the corresponding SDE. To perform this factorisation, let us consider the contribution of each noise independently. We first set $\sigma_{2}=\sigma_{3}=0$ to keep only $v_{1}$. The resulting matrix $\tilde{c}$ is of rank 3 , while the original matrix $b b^{\mathrm{T}}$ was of rank 1 . This means that the noise $v_{1}$ gave rise to 3 independent Gaussian white noises, all controlled by the same variance $\sigma_{1}$. We can factorise this matrix for $\sigma_{2}=$ $\sigma_{3}=0$ as $\tilde{c}=\tilde{b}_{1} \tilde{b}_{1}^{\mathrm{T}}$, where

$$
\tilde{b}_{1}=\frac{\sqrt{\sigma_{1}}}{2 k}\left(\begin{array}{ccc}
-\cos ^{2} \theta & -\frac{\cos ^{2} \theta \cos \left(2 \phi_{1}\right)}{\sqrt{2}} & \sqrt{2} \cos ^{2} \theta \cos \phi_{1} \sin \phi_{1} \\
-\sin ^{2} \theta & -\frac{\sin ^{2} \theta \cos \left(2 \phi_{2}\right)}{\sqrt{2}} & \sqrt{2} \sin ^{2} \theta \cos \phi_{2} \sin \phi_{2} \\
\frac{\cot \left(\phi_{1}-\phi_{2}\right) \sin (2 \theta)}{2} & \frac{\cos \left(\phi_{1}+\phi_{2}\right) \sin (2 \theta)}{2 \sqrt{2} \sin \left(\phi_{1}-\phi_{2}\right)} & -\frac{\sin \left(\phi_{1}+\phi_{2}\right) \sin (2 \theta)}{2 \sqrt{2} \sin \left(\phi_{1}-\phi_{2}\right)}
\end{array}\right) \text {. }
$$


The same situation occurs for the noises $v_{2}$ and $v_{3}$, which each give rise to three new independent noises. Similarly, we obtain a matrix $\tilde{b}_{2}$ for $\sigma_{1}=\sigma_{3}=0$ and $\tilde{b}_{3}$ for $\sigma_{1}=\sigma_{2}=0$. Finally, we can write the full matrix $\tilde{c}=\tilde{b} \tilde{b}^{\mathrm{T}}$, where $\tilde{b}$ is the following $3 \times 9$ matrix, with block structure

$$
\tilde{b}=\left(\begin{array}{lll}
\tilde{b}_{1} & \tilde{b}_{2} & \tilde{b}_{3}
\end{array}\right) .
$$

We can then rewrite the Fokker-Planck equation (B.9) in the form

$$
\frac{\partial P}{\partial x}\left(\tilde{\phi}_{1}, \tilde{\phi}_{2}, \theta\right)=\frac{1}{2} \sum_{i, j=1}^{3} \sum_{p=1}^{9} \frac{\partial}{\partial X_{i}}\left(\tilde{b}_{i p} \frac{\partial}{\partial X_{j}} \tilde{b}_{j p}\right) P,
$$

where the drift terms have cancelled out with the terms coming from the derivative of the matrix $\tilde{b}$. We can write the corresponding SDE by introducing three new independent matrices of white noises, which we denote $\tilde{V}_{i}, i=1,2,3$. Rewriting the result in terms of

$$
\widetilde{\mathcal{S}}=\mathcal{O}^{\mathrm{T}} \operatorname{Diag}\left(\mathrm{e}^{2 \mathrm{i} \tilde{\phi}_{1}}, \mathrm{e}^{2 \mathrm{i} \tilde{\phi}_{2}}\right) \mathcal{O}=\mathrm{e}^{-2 \mathrm{i} k x} \mathcal{S}
$$

gives

$$
\partial_{x} \widetilde{\mathcal{S}}=\frac{1}{2 \mathrm{i} k}\left\{V_{1}+\widetilde{\mathcal{S}} V_{1} \widetilde{\mathcal{S}}-\mathrm{i}\left(V_{2}-\widetilde{\mathcal{S}} V_{2} \widetilde{\mathcal{S}}\right)+V_{3} \widetilde{\mathcal{S}}+\widetilde{\mathcal{S}} V_{3}\right\}, \quad \text { (Stratonovich) }
$$

where

$$
V_{1} \stackrel{(\text { law })}{=} V_{2} \stackrel{(\text { law })}{=} \frac{1}{\sqrt{2}} V, \quad V_{3} \stackrel{(\text { law })}{=} V, \quad V_{i} \text { and } V_{j} \text { independent. }
$$

\section{ORCID iDs}

Aurélien Grabsch (1D https://orcid.org/0000-0003-4316-5190

Christophe Texier (D) https://orcid.org/0000-0003-4987-5150

\section{References}

[1] Verbaarschot J J M, Weidenmüller H A and Zirnbauer M R 1985 Grassmann integration in stochastic quantum physics: the case of compound-nucleus scattering Phys. Rep. 129 367-438

[2] Mitchell G E, Richter A and Weidenmüller H A 2010 Random matrices and chaos in nuclear physics: Nuclear reactions Rev. Mod. Phys. 82 2845-901

[3] Guhr T, Müller-Groeling A and Weidenmüller H A 1998 Random-matrix theories in quantum physics: common concepts Phys. Rep. 299 189-425

[4] Sebbah P and Genack A 1998 Multiple scattering of microwaves New Aspects of Electromagnetic and Acoustic Wave Diffusion vol 144 (Berlin: Springer) pp 28-34

[5] Beenakker C W J 1997 Random-matrix theory of quantum transport Rev. Mod. Phys. 69 731-808

[6] Mello P A and Kumar N 2004 Quantum Transport in Mesoscopic Systems-Complexity and Statistical Fluctuations (Oxford: Oxford University Press)

[7] Wigner E P 1955 Lower limit for the energy derivative of the scattering phase shift Phys. Rev. 98 $145-7$

[8] Smith F T 1960 Lifetime matrix in collision theory Phys. Rev. 118 349-56

[9] de Carvalho C A A and Nussenzveig H M 2002 Time delay Phys. Rep. 364 83-174

[10] Texier C 2016 Wigner time delay and related concepts-pplication to transport in coherent conductors Physica E 82 16-33 (see arXiv:1507.00075 [cond-mat] for an updated version)

[11] Brouwer P W 1995 Generalized circular ensemble of scattering matrices for a chaotic cavity with nonideal leads Phys. Rev. B 51 16878-84 
[12] Fyodorov Y V and Sommers H-J 1997 Statistics of resonance poles, phase shifts and time delays in quantum chaotic scattering: random matrix approach for systems with broken time-reversal invariance J. Math. Phys. 38 1918-81

[13] Blümel R and Smilansky U 1988 Classical irregular scattering and its quantum-mechanical implications Phys. Rev. Lett. 60 477-80

[14] Mello P A, Pereyra P and Seligman T H 1985 Information theory and statistical nuclear reactions. I. General theory and applications to few-channel problems Ann. Phys., NY 161 254-75

[15] Mehta M L 2004 Random Matrices 3rd edn (New York: Elsevier)

[16] Brouwer P W and Büttiker M 1997 Charge-relaxation and dwell time in the fluctuating admittance of a chaotic cavity Europhys. Lett. 37 441-6

[17] Brouwer P W, Frahm K M and Beenakker C W 1997 Quantum mechanical time-delay matrix in chaotic scattering Phys. Rev. Lett. 784737

[18] Brouwer P W, Frahm K M and Beenakker C W 1999 Distribution of the quantum mechanical timedelay matrix for a chaotic cavity Waves Random Media 9 91-104

[19] Mezzadri F and Simm N J $2013 \tau$-function theory of quantum chaotic transport with $\beta=1,2,4$ Commun. Math. Phys. 324 465-513

[20] Texier C and Majumdar S N 2013 Wigner time-delay distribution in chaotic cavities and freezing transition Phys. Rev. Lett. 110250602

Texier C and Majumdar S N 2014112139902

[21] Mezzadri F and Simm N J 2011 Moments of the transmission eigenvalues, proper delay times, and random matrix theory. I J. Math. Phys. 52103511

[22] Mezzadri F and Simm N J 2012 Moments of the transmission eigenvalues, proper delay times, and random matrix theory. II J. Math. Phys. 53053504

[23] Grabsch A and Texier C 2015 Capacitance and charge relaxation resistance of chaotic cavities-Joint distribution of two linear statistics in the Laguerre ensemble of random matrices Europhys. Lett. 10950004

[24] Cunden F D 2015 Statistical distribution of the Wigner-Smith time-delay matrix moments for chaotic cavities Phys. Rev. E 91060102

[25] Cunden F D, Mezzadri F, Simm N and Vivo P 2016 Correlators for the Wigner-Smith time-delay matrix of chaotic cavities J. Phys. A: Math. Theor. 49 18LT01

[26] Marciani M, Schomerus H and Beenakker C W J 2016 Effect of a tunnel barrier on the scattering from a Majorana bound state in an Andreev billiard Physica E 77 54-64

[27] Grabsch A, Savin D V and Texier C 2018 Wigner-Smith time-delay matrix in chaotic cavities with non-ideal contacts J. Phys. A: Math. Theor. 51404001

[28] Grabsch A 2020 Distribution of the Wigner-Smith time-delay matrix for chaotic cavities with absorption and coupled Coulomb gases J. Phys. A: Math. Theor. $\mathbf{5 3} 025202$

[29] Texier C and Comtet A 1999 Universality of the Wigner time delay distribution for one-dimensional random potentials Phys. Rev. Lett. 82 4220-3

[30] Faris W G and Tsay W J 1994 Time delay in random scattering SIAM J. Appl. Math. 54 443-55

[31] Comtet A and Texier C 1997 On the distribution of the Wigner time delay in one-dimensional disordered systems J. Phys. A: Math. Gen. 30 8017-25

[32] Monthus C and Comtet A 1994 On the flux distribution in a one-dimensional disordered system $J$. Physique I 4 635-53

[33] Comtet A, Monthus C and Yor M 1998 Exponential functionals of Brownian motion and disordered systems J. Appl. Probab. 35255

[34] Ossipov A, Kottos T and Geisel T 2000 Statistical properties of phases and delay times of the onedimensional Anderson model with one open channel Phys. Rev. B 61 11411-5

[35] Yor M 2000 Exponential Functionals of Brownian Motion and Related Processes (Berlin: Springer)

[36] Matsumoto H and Yor M 2005 Exponential functionals of Brownian motion, I: probability laws at fixed time Probab. Surv. 2 312-47

[37] Matsumoto $\mathrm{H}$ and Yor M 2005 Exponential functionals of Brownian motion, II: some related diffusion processes Probab. Surv. $2348-84$

[38] Monthus C 1995 Étude de quelques fonctionnelles du mouvement brownien et de certaines propriétés de la diffusion unidimensionnelle en milieu aléatoire $P h D$ Thesis (Université Paris) 6

Monthus C 1995 Etude de quelques fonctionnelles du mouvement brownien et de certaines propriétés de la diffusion unidimensionnelle en milieu aléatoire Ann. Phys., Paris 20341 
[39] Comtet A, Desbois J and Texier C 2005 Functionals of the Brownian motion, localization and metric graphs J. Phys. A: Math. Gen. 38 R341-83

[40] Dufresne D 1990 The distribution of a perpetuity, with application to risk theory and pension funding Scand. Actuar. J. 39-79

[41] Texier C 1999 Quelques aspects du transport quantique dans les systèmes désordonnés de basse dimension PhD Thesis (Université Paris) 6

[42] Dunlap D H, Wu H-L and Phillips P 1990 Absence of localization in a random-dimer model Phys. Rev. Lett. $\mathbf{6 5} 88$

[43] Ossipov A and Fyodorov Y V 2005 Statistics of delay times in mesoscopic systems as a manifestation of eigenfunction fluctuations Phys. Rev. B 71125133

[44] Kottos T 2005 Statistics of resonances and delay times in random media: beyond random matrix theory J. Phys. A: Math. Theor. 38 10761-86

[45] Ossipov A 2018 Scattering approach to Anderson localisation Phys. Rev. Lett. 121076601

[46] Beenakker C W J 2001 Dynamics of localization in a waveguide Photonic Crystals and Light Localization in the 21st Century NATO (Science Series C563) ed C Soukoulis (Dordrecht: Kluwer) pp 489-508

[47] Beenakker C W J and Brouwer P W 2001 Distribution of the reflection eigenvalues of a weakly absorbing chaotic cavity Physica E 9 463-6

[48] Grabsch A and Texier C 2016 Distribution of spectral linear statistics on random matrices beyond the large deviation function-Wigner time delay in multichannel disordered wires J. Phys. A: Math. Theor. 49465002

[49] Dorokhov O N 1982 Transmission coefficient and the localization length of an electron in $N$ bound disordered chains JETP Lett. 36 318-21

[50] Dorokhov O N 1988 Solvable model of multichannel localization Phys. Rev. B 37 10526-41

[51] Halperin B I 1965 Green's functions for a particle in a one-dimensional random potential Phys. Rev. 139 A $104-17$

[52] Akkermans E and Montambaux G 2007 Mesoscopic Physics of Electrons and Photons (Cambridge: Cambridge University Press)

[53] Texier C 2015 Mécanique Quantique 2nd edn (Paris: Dunod)

[54] Antsygina T N, Pastur L A and Slyusarev V A 1981 Localization of states and kinetic properties of one-dimensional disordered systems Sov. J. Low Temp. Phys. 7 1-21

[55] Cohen A, Roth Y and Shapiro B 1988 Universal distributions and scaling in disordered systems Phys. Rev. B 38 12125-32

[56] Texier C 2020 Generalized Lyapunov exponent of random matrices and universality classes for SPS in 1D Anderson localisation Europhys. Lett. 13117002

[57] Le Jan Y 1985 On isotropic Brownian motion Z. Wahrscheinlichkeitstheor. Verwandte Geb. 70 609-20

[58] Newman C M 1986 The distribution of Lyapunov exponents: exact results for random matrices Commun. Math. Phys. 103 121-6

[59] Grabsch A 2018 Random matrices in statistical physics: quantum scattering and disordered systems PhD Thesis (Université Paris Saclay)

[60] Rider B and Valkó B 2015 Matrix Dufresne identities Int. Math. Res. Not. 2016 174-218

[61] Grabsch A and Texier C 2016 Topological phase transitions in the 1D multichannel Dirac equation with random mass and a random matrix model Europhys. Lett. 11617004

[62] Friedel J 1958 Metallic alloys Nuovo Cimento, Suppl. 7 287-311

[63] Texier C and Büttiker M 2003 Local Friedel sum rule in graphs Phys. Rev. B 67245410

[64] Texier C and Degiovanni P 2003 Charge and current distribution in graphs J. Phys. A: Math. Gen. 36 12425-52

[65] Texier C 2002 Scattering theory on graphs (2): the Friedel sum rule J. Phys. A: Math. Gen. 35 3389-407 